\title{
Stability and Hopf Bifurcation Analysis for a Computer Virus Propagation Model with Two Delays and Vaccination
}

\author{
Zizhen Zhang, ${ }^{1}$ Yougang Wang, ${ }^{1}$ Dianjie Bi, ${ }^{1}$ and Luca Guerrini ${ }^{2}$ \\ ${ }^{1}$ School of Management Science and Engineering, Anhui University of Finance and Economics, Bengbu 233030, China \\ ${ }^{2}$ Department of Management, Marche Polytechnic University, Piazza Martelli 8, 60121 Ancona, Italy \\ Correspondence should be addressed to Zizhen Zhang; zzzhaida@163.com
}

Received 5 January 2017; Accepted 23 February 2017; Published 20 March 2017

Academic Editor: Lu-Xing Yang

Copyright (c) 2017 Zizhen Zhang et al. This is an open access article distributed under the Creative Commons Attribution License, which permits unrestricted use, distribution, and reproduction in any medium, provided the original work is properly cited.

\begin{abstract}
A further generalization of an SEIQRS-V (susceptible-exposed-infectious-quarantined-recovered-susceptible with vaccination) computer virus propagation model is the main topic of the present paper. This paper specifically analyzes effects on the asymptotic dynamics of the computer virus propagation model when two time delays are introduced. Sufficient conditions for the asymptotic stability and existence of the Hopf bifurcation are established by regarding different combination of the two delays as the bifurcation parameter. Moreover, explicit formulas that determine the stability, direction, and period of the bifurcating periodic solutions are obtained with the help of the normal form theory and center manifold theorem. Finally, numerical simulations are employed for supporting the obtained analytical results.
\end{abstract}

\section{Introduction}

Computer viruses, including conventional viruses and network worms, can propagate among computers with no human awareness and popularization of Internet has been the major propagation channel of viruses $[1,2]$. The past few decades have witnessed the great financial losses caused by computer viruses. Therefore, it is of considerable importance to investigate the laws describing propagation of computer viruses in order to provide some help with preventing computer viruses. For that purpose and in view of the fact that propagation of computer viruses among computers resembles that of biological viruses among a population, many dynamical models describing propagation of computer viruses across the Internet have been established by the scholars at home and abroad, such as conventional models [3-8], stochastic models [9-12], and delayed models [13-18]. There are also some other computer virus models [19-21] combined with network theory to investigate the impact of the network topology, the patch forwarding, and the network eigenvalue on the viral prevalence.

As is known, vaccination is regarded as one of the most effective measures of preventing computer viruses and the awareness that there exist many infected computers would enhance the probability that the user of a susceptible computer will make his computer vaccinated [22, 23]. However, the mentioned models above neglect the influence of vaccination strategy on the propagation of computer viruses. Recently, considering the importance of vaccination, Kumar et al. [24] proposed the following SEIQRS-V computer virus propagation model:

$$
\begin{aligned}
\frac{d S(t)}{d t}= & A-\beta S(t) I(t)-d S(t)-\rho S(t)+\theta R(t) \\
& +\chi V(t), \\
\frac{d E(t)}{d t}= & \beta S(t) I(t)-d E(t)-\gamma E(t), \\
\frac{d I(t)}{d t}= & \gamma E(t)-d I(t)-\alpha I(t)-\delta I(t)-\eta I(t), \\
\frac{d Q(t)}{d t}= & \delta I(t)-d Q(t)-\alpha Q(t)-\varepsilon Q(t), \\
\frac{d R(t)}{d t}= & \varepsilon Q(t)-d R(t)-\theta R(t)+\eta I(t), \\
\frac{d V(t)}{d t}= & \rho S(t)-d V(t)-\chi V(t),
\end{aligned}
$$


where $S(t), E(t), I(t), Q(t), R(t)$, and $V(t)$ denote the numbers of the uninfected computers, the exposed computers, the infected computers, the quarantined computers, recovered computers, and vaccinated computers at time $t$, respectively. $A$ is the birth rate of new computers in the network; $d$ is the death rate of the computers due to the reason other than the attack of viruses; $\alpha$ is the death rate of computers due to the attack of viruses; $\beta$ is the contact rate of the uninfected computers; $\rho, \theta, \chi, \gamma, \delta, \eta$, and $\varepsilon$ are the transition rates between the states in system (1).

Obviously, system (1) neglects the delays in the procedure of viruses' propagation and it is investigated under the assumption that the transition between the states is instantaneous. This is not reasonable with reality. For example, it needs a period to clean the viruses in the infected and quarantined computers for antivirus software and there is usually a temporary immunity period for the recovered and the vaccinated computers because of the effect of the antivirus software. In addition, a stability switch occurs even when an ignored delay is small for a dynamical system. Based on this, we introduce two delays into system (1) and get the following delayed system:

$$
\begin{aligned}
\frac{d S(t)}{d t}= & A-\beta S(t) I(t)-d S(t)-\rho S(t)+\theta R\left(t-\tau_{2}\right) \\
& +\chi V\left(t-\tau_{2}\right), \\
\frac{d E(t)}{d t}= & \beta S(t) I(t)-d E(t)-\gamma E(t), \\
\frac{d I(t)}{d t}= & \gamma E(t)-d I(t)-\alpha I(t)-\delta I(t)-\eta I\left(t-\tau_{1}\right), \\
\frac{d Q(t)}{d t}= & \delta I(t)-d Q(t)-\alpha Q(t)-\varepsilon Q\left(t-\tau_{1}\right), \\
\frac{d R(t)}{d t}= & \varepsilon Q\left(t-\tau_{1}\right)-d R(t)-\theta R\left(t-\tau_{2}\right) \\
& +\eta I\left(t-\tau_{1}\right), \\
\frac{d V(t)}{d t}= & \rho S(t)-d V(t)-\chi V\left(t-\tau_{2}\right),
\end{aligned}
$$

where $\tau_{1}$ is the time delay due to the period that antivirus software uses to clean the viruses in the infected and quarantined computers and $\tau_{2}$ is the time delay due to the temporary immunity period of the recovered and the vaccinated computers.

To the best of our knowledge, until now, there is no good analysis on system (2). Therefore, it is meaningful to analyze the proposed system with two delays.

The rest of this paper is organized as follows. In the next section, we analyze the threshold of Hopf bifurcation of system (2) by regarding different combination of the two delays as the bifurcation parameter. In Section 3, by means of the normal form theory and center manifold theorem, direction and stability of the Hopf bifurcation for $\tau_{1}>0$ and $\tau_{2}>0$ are investigated. Simulation results of system (2) are shown in Section 4. Finally, we finish the paper with conclusions in Section 5.

\section{Analysis of Hopf Bifurcation}

By direct computation, we know that if $A R_{0}(d+\chi)>d^{2}+$ $(\rho+\chi) d$ and $\beta(d+\theta)(d+\alpha+\varepsilon)>R_{0} \theta \varepsilon \delta+R_{0} \theta \eta(d+$ $\alpha+\varepsilon)$, then system (2) has a unique viral equilibrium $P_{*}\left(S_{*}, E_{*}, I_{*}, Q_{*}, R_{*}, V_{*}\right)$, where

$$
\begin{aligned}
S_{*} & =\frac{(d+\gamma)(d+\alpha+\delta+\eta)}{\beta \gamma}=\frac{1}{R_{0}}, \\
E_{*} & =\frac{d+\alpha+\delta+\eta}{\gamma} I_{*}, \\
R_{*} & =\frac{\varepsilon \delta+\eta(d+\alpha+\varepsilon)}{(d+\theta)(d+\alpha+\varepsilon)} I_{*}, \\
V_{*} & =\frac{\rho}{(d+\chi) R_{0}}, \\
Q_{*} & =\frac{\delta}{d+\alpha+\varepsilon} I_{*}, \\
I_{*} & \\
= & \frac{(d+\theta)(d+\alpha+\varepsilon)\left[d^{2}+(\rho+\chi) d-A R_{0}(d+\chi)\right]}{(d+\chi)\left[R_{0} \theta \varepsilon \delta+(d+\alpha+\varepsilon)\left(R_{0} \theta \eta-\beta d-\beta \theta\right)\right]}, \\
R_{0} & =\frac{\beta \gamma}{(d+\gamma)(d+\alpha+\delta+\eta)} .
\end{aligned}
$$

The linearized section of system (2) at $P_{*}\left(S_{*}, E_{*}, I_{*}, Q_{*}\right.$, $\left.R_{*}, V_{*}\right)$ is as follows:

$$
\begin{aligned}
\frac{d S(t)}{d t}= & a_{1} S(t)+a_{2} I(t)+c_{1} R\left(t-\tau_{2}\right)+c_{2} V\left(t-\tau_{2}\right), \\
\frac{d E(t)}{d t}= & a_{3} S(t)+a_{4} E(t)+a_{5} I(t), \\
\frac{d I(t)}{d t}= & a_{6} E(t)-a_{7} I(t)+b_{1} I\left(t-\tau_{1}\right), \\
\frac{d Q(t)}{d t}= & a_{8} I(t)+a_{9} Q(t)+b_{2} Q\left(t-\tau_{1}\right), \\
\frac{d R(t)}{d t}= & a_{10} R(t)+b_{3} I\left(t-\tau_{1}\right)+b_{4} Q\left(t-\tau_{1}\right) \\
& +c_{3} R\left(t-\tau_{2}\right), \\
\frac{d V(t)}{d t}= & a_{11} S(t)+a_{12} V(t)+c_{4} V\left(t-\tau_{2}\right),
\end{aligned}
$$

where

$$
\begin{aligned}
& a_{1}=-\left(\beta I_{*}+d+\rho\right), \\
& a_{2}=-\beta S_{*}, \\
& a_{3}=\beta I_{*}, \\
& a_{4}=-(d+\gamma),
\end{aligned}
$$




$$
\begin{aligned}
& a_{5}=\beta S_{*}, \\
& a_{6}=\gamma, \\
& a_{7}=-(d+\alpha+\delta), \\
& a_{8}=\delta, \\
& a_{9}=-(d+\alpha), \\
& a_{10}=-d, \\
& a_{11}=\rho, \\
& a_{12}=-d, \\
& b_{1}=-\eta, \\
& b_{2}=-\varepsilon, \\
& b_{3}=\eta, \\
& b_{4}=\varepsilon, \\
& c_{1}=\theta, \\
& c_{2}=\chi, \\
& c_{3}=-\theta, \\
& c_{4}=-\chi .
\end{aligned}
$$

Then, the characteristic equation for system (4) can be obtained:

$$
\begin{aligned}
\lambda^{6}+ & A_{5} \lambda^{5}+A_{4} \lambda^{4}+A_{3} \lambda^{3}+A_{2} \lambda^{2}+A_{1} \lambda+A_{0} \\
& +\left(B_{5} \lambda^{5}+B_{4} \lambda^{4}+B_{3} \lambda^{3}+B_{2} \lambda^{2}+B_{1} \lambda+B_{0}\right) e^{-\lambda \tau_{1}} \\
& +\left(C_{5} \lambda^{5}+C_{4} \lambda^{4}+C_{3} \lambda^{3}+C_{2} \lambda^{2}+C_{1} \lambda+C_{0}\right) e^{-\lambda \tau_{2}} \\
& +\left(D_{4} \lambda^{4}+D_{3} \lambda^{3}+D_{2} \lambda^{2}+D_{1} \lambda+D_{0}\right) e^{-\lambda\left(\tau_{1}+\tau_{2}\right)} \\
& +\left(E_{3} \lambda^{3}+E_{2} \lambda^{2}+E_{1} \lambda+E_{0}\right) e^{-\lambda\left(\tau_{1}+2 \tau_{2}\right)} \\
& +\left(F_{4} \lambda^{4}+F_{3} \lambda^{3}+F_{2} \lambda^{2}+F_{1} \lambda+F_{0}\right) e^{-2 \lambda \tau_{1}} \\
& +\left(G_{4} \lambda^{4}+G_{3} \lambda^{3}+G_{2} \lambda^{2}+G_{1} \lambda+G_{0}\right) e^{-2 \lambda \tau_{2}} \\
& +\left(H_{3} \lambda^{3}+H_{2} \lambda^{2}+H_{1} \lambda+H_{0}\right) e^{-\lambda\left(2 \tau_{1}+\tau_{2}\right)} \\
& +\left(I_{2} \lambda^{2}+I_{1} \lambda+I_{0}\right) e^{-2 \lambda\left(\tau_{1}+\tau_{2}\right)}=0
\end{aligned}
$$

with

$$
\begin{aligned}
A_{0} & =a_{9} a_{12}\left(a_{1} a_{4} a_{7} a_{10}+a_{2} a_{3} a_{6} a_{10}-a_{1} a_{5} a_{6} a_{10}\right. \\
& \left.-a_{3} a_{6} b_{3} c_{1}\right),
\end{aligned}
$$

$$
\begin{aligned}
& A_{1}=a_{5} a_{6}\left(a_{1} a_{9}\left(a_{10}+a_{12}\right)+a_{10} a_{12}\left(a_{1}+a_{9}\right)\right) \\
& +a_{3} a_{6} b_{3} c_{1}\left(a_{9}+a_{12}\right)-a_{2} a_{3} a_{6}\left(a_{9} a_{10}+a_{9} a_{12}\right. \\
& \left.+a_{10} a_{12}\right)-a_{1} a_{4} a_{7} a_{9}\left(a_{10}+a_{12}\right) \\
& -a_{10} a_{12}\left(a_{1} a_{4}\left(a_{7}+a_{9}\right)+a_{7} a_{9}\left(a_{1}+a_{4}\right)\right) \text {, } \\
& A_{2}=a_{2} a_{3} a_{6}\left(a_{9}+a_{10}+a_{12}\right)+a_{1} a_{4} a_{7} a_{9}-a_{3} a_{6} b_{3} c_{1} \\
& -a_{5} a_{6}\left(a_{1} a_{9}+a_{10} a_{12}+\left(a_{1}+a_{9}\right)\left(a_{10}+a_{12}\right)\right) \\
& +a_{10} a_{12}\left(a_{1} a_{4}+a_{7} a_{9}+\left(a_{1}+a_{4}\right)\left(a_{7}+a_{9}\right)\right)+\left(a_{10}\right. \\
& \left.+a_{12}\right)\left(a_{1} a_{4}\left(a_{7}+a_{9}\right)+a_{7} a_{9}\left(a_{1}+a_{4}\right)\right), \\
& A_{3}=a_{5} a_{6}\left(a_{1}+a_{9}+a_{10}+a_{12}\right)-a_{2} a_{3} a_{6}-a_{1} a_{4}\left(a_{7}\right. \\
& \left.+a_{9}\right)-a_{7} a_{9}\left(a_{1}+a_{4}\right)-a_{10} a_{12}\left(a_{1}+a_{4}+a_{7}+a_{9}\right) \\
& -\left(a_{10}+a_{12}\right)\left(a_{1} a_{4}+a_{7} a_{9}+\left(a_{1}+a_{4}\right)\left(a_{7}+a_{9}\right)\right), \\
& A_{4}=a_{1} a_{4}+a_{7} a_{9}+a_{10} a_{12}-a_{5} a_{6}+\left(a_{1}+a_{4}\right)\left(a_{7}+a_{9}\right) \\
& +\left(a_{10}+a_{12}\right)\left(a_{1}+a_{4}+a_{7}+a_{9}\right), \\
& A_{5}=-\left(a_{1}+a_{4}+a_{7}+a_{9}+a_{10}+a_{12}\right) \text {, } \\
& B_{0}=a_{1} a_{4} a_{10} a_{12}\left(a_{7} b_{2}+a_{9} b_{1}\right)-a_{6} a_{10} a_{12} b_{2}\left(a_{1} a_{5}\right. \\
& \left.+a_{2} a_{3}\right) \text {, } \\
& B_{1}=a_{5} a_{6} b_{2}\left(a_{1} a_{10}+a_{1} a_{12}+a_{10} a_{12}\right)-a_{2} a_{3} a_{6} b_{2}\left(a_{10}\right. \\
& \left.+a_{12}\right)-\left(a_{7} b_{2}+a_{9} b_{1}\right)\left(a_{1} a_{4}\left(a_{10}+a_{12}\right)\right. \\
& \left.+a_{10} a_{12}\left(a_{1}+a_{4}\right)\right) \\
& -a_{1} a_{4} a_{10} a_{12}\left(b_{1}+b_{2}\right) \\
& B_{2}=\left(a_{7} b_{2}+a_{9} b_{1}\right)\left(a_{1} a_{4}+a_{10} a_{12}\right. \\
& \left.+\left(a_{1}+a_{4}\right)\left(a_{10}+a_{12}\right)\right)+a_{2} a_{3} a_{6} b_{2}+\left(b_{1}+b_{2}\right) \\
& \cdot\left(a_{1} a_{4}\left(a_{10}+a_{12}\right)+a_{10} a_{12}\left(a_{1}+a_{4}\right)\right) \text {. } \\
& B_{3}=a_{5} a_{6} b_{2}-\left(a_{7} b_{2}+a_{9} b_{1}\right)\left(a_{1}+a_{4}+a_{10}+a_{12}\right)-\left(b_{1}\right. \\
& \left.+b_{2}\right)\left(a_{1} a_{4}+a_{10} a_{12}+\left(a_{1}+a_{4}\right)\left(a_{10}+a_{12}\right)\right) \text {, } \\
& B_{4}=a_{7} b_{2}+a_{9} b_{1}+\left(b_{1}+b_{2}\right)\left(a_{1}+a_{4}+a_{10}+a_{12}\right) \text {, } \\
& B_{5}=-\left(b_{1}+b_{2}\right) \text {, } \\
& C_{0}=a_{1} a_{4} a_{7} a_{9}\left(a_{10} c_{4}+a_{12} c_{3}\right)+a_{9} a_{10} a_{11} c_{2}\left(a_{4} a_{7}\right. \\
& \left.-a_{5} a_{6}\right)+a_{6} a_{9}\left(a_{10} c_{4}+a_{12} c_{3}\right)\left(a_{2} a_{3}-a_{1} a_{5}\right), \\
& C_{1}=a_{9}\left(c_{3}+c_{4}\right)\left(a_{1} a_{5} a_{6}-a_{2} a_{3} a_{6}-a_{1} a_{4} a_{7}\right) \\
& +a_{5} a_{6} a_{11} c_{2}\left(a_{9}+a_{10}\right)+a_{6}\left(a_{1} a_{5}-a_{2} a_{3}+a_{5} a_{9}\right) \\
& \cdot\left(a_{10} c_{4}+a_{12} c_{3}\right)-a_{11} c_{2}\left(a_{4} a_{7}\left(a_{9}+a_{10}\right)\right. \\
& \left.+a_{9} a_{10}\left(a_{4}+a_{7}\right)\right) \text {, }
\end{aligned}
$$




$$
\begin{aligned}
& C_{2}=\left(c_{3}+c_{4}\right)\left(a_{1} a_{4}\left(a_{7}+a_{9}\right)+a_{7} a_{9}\left(a_{1}+a_{4}\right)\right) \\
& +\left(a_{10} c_{4}+a_{12} c_{3}\right)\left(a_{1} a_{4}+a_{7} a_{9}+\left(a_{1}+a_{4}\right)\left(a_{7}+a_{9}\right)\right) \\
& -a_{5} a_{6}\left(a_{10} c_{4}+a_{12} c_{3}-a_{11} c_{2}\right)+a_{6}\left(c_{3}+c_{4}\right)\left(a_{2} a_{3}\right. \\
& \left.-a_{1} a_{5}-a_{5} a_{9}\right)+a_{11} c_{2}\left(a_{4} a_{7}+a_{9} a_{10}\right. \\
& \left.+\left(a_{4}+a_{7}\right)\left(a_{9}+a_{10}\right)\right) \text {, } \\
& C_{3}=a_{5} a_{6}\left(c_{3}+c_{4}\right)-a_{11} c_{2}\left(a_{4}+a_{7}+a_{9}+a_{10}\right)-\left(a_{10} c_{4}\right. \\
& \left.+a_{12} c_{3}\right)\left(a_{1}+a_{4}+a_{7}+a_{9}\right)-\left(c_{3}+c_{4}\right)\left(a_{1} a_{4}+a_{7} a_{9}\right. \\
& \left.+\left(a_{1}+a_{4}\right)\left(a_{7}+a_{9}\right)\right) \text {, } \\
& C_{4}=a_{10} c_{4}+a_{11} c_{2}+a_{12} c_{3}+\left(c_{3}+c_{4}\right)\left(a_{1}+a_{4}+a_{7}\right. \\
& \left.+a_{9}\right) \\
& C_{5}=-\left(c_{3}+c_{4}\right) \text {, } \\
& D_{0}=a_{4}\left(a_{1} a_{10} c_{4}+a_{1} a_{12} c_{3}+a_{10} a_{11} c_{2}\right)\left(a_{7} b_{2}+a_{9} b_{1}\right) \\
& +a_{6}\left(a_{3} a_{8} a_{12} b_{4} c_{4}-a_{5} a_{10} a_{11} b_{2} c_{2}\right)-a_{6} b_{2}\left(a_{1} a_{5}\right. \\
& \left.-a_{2} a_{3}\right)\left(a_{10} c_{4}+a_{12} c_{3}\right) \\
& D_{1}=\left(c_{3}+c_{4}\right)\left(a_{5} b_{2}\left(a_{1} a_{5}-a_{2} a_{3}\right)-a_{1} a_{4}\left(a_{7} b_{2}+a_{9} b_{1}\right)\right) \\
& +a_{6}\left(a_{5} a_{11} b_{2} c_{2}-a_{3} a_{8} b_{4} c_{4}\right)+a_{5} a_{6} b_{2}\left(a_{10} c_{4}+a_{12} c_{3}\right) \\
& -a_{11} c_{2}\left(a_{4} a_{10}\left(b_{1}+b_{2}\right)+\left(a_{4}+a_{10}\right)\left(a_{7} b_{2}+a_{9} b_{1}\right)\right) \\
& -\left(a_{10} c_{4}+a_{12} c_{3}\right)\left(a_{1} a_{4}\left(b_{1}+b_{2}\right)\right. \\
& \left.+\left(a_{1}+a_{4}\right)\left(a_{7} b_{2}+a_{9} b_{1}\right)\right) \text {, } \\
& D_{2}=\left(a_{10} c_{4}+a_{12} c_{3}\right)\left(a_{7} b_{2}+a_{9} b_{1}+\left(b_{1}+b_{2}\right)\left(a_{1}+a_{4}\right)\right) \\
& +\left(c_{3}+c_{4}\right)\left(a_{1} a_{4}\left(b_{1}+b_{2}\right)+\left(a_{1}+a_{4}\right)\left(a_{7} b_{2}+a_{9} b_{1}\right)\right) \\
& +a_{11} c_{2}\left(a_{7} b_{2}+a_{9} b_{1}+\left(a_{4}+a_{10}\right)\left(b_{1}+b_{2}\right)\right) \\
& -a_{5} a_{6} b_{2}\left(c_{3}+c_{4}\right) \\
& D_{3}=-\left(c_{3}+c_{4}\right)\left(a_{7} b_{2}+a_{9} b_{1}+\left(b_{1}+b_{2}\right)\left(a_{1}+a_{4}\right)\right) \\
& -\left(b_{1}+b_{2}\right)\left(a_{10} c_{4}+a_{11} c_{2}+a_{12} c_{3}\right) \text {, } \\
& D_{4}=\left(b_{1}+b_{2}\right)\left(c_{3}+c_{4}\right) \text {, } \\
& E_{0}=a_{4} c_{3}\left(a_{7} b_{2}+a_{9} b_{1}\right)\left(a_{1} c_{4}+a_{11} c_{2}\right)+a_{6} b_{2} c_{3} c_{4}\left(a_{2} a_{3}\right. \\
& \left.-a_{1} a_{5}\right)-a_{6}\left(a_{3} a_{9} b_{3} c_{1} c_{4}+a_{5} a_{11} b_{2} c_{2} c_{3}\right), \\
& E_{1}=a_{6} c_{4}\left(a_{5} b_{2} c_{3}+a_{3} b_{3} c_{1}\right)-a_{11} c_{2} c_{3}\left(b_{1}\left(a_{4}+a_{9}\right)\right. \\
& \left.+b_{2}\left(a_{4}+a_{7}\right)\right)-c_{3} c_{4}\left(a_{1} a_{4}\left(b_{1}+b_{2}\right)\right. \\
& \left.+\left(a_{1}+a_{4}\right)\left(a_{7} b_{2}+a_{9} b_{1}\right)\right), \\
& E_{2}=c_{3} c_{4}\left(a_{7} b_{2}+a_{9} b_{1}+\left(a_{1}+a_{4}\right)\left(b_{1}+b_{2}\right)\right) \\
& +a_{11} c_{2} c_{3}\left(b_{1}+b_{2}\right) \text {, }
\end{aligned}
$$

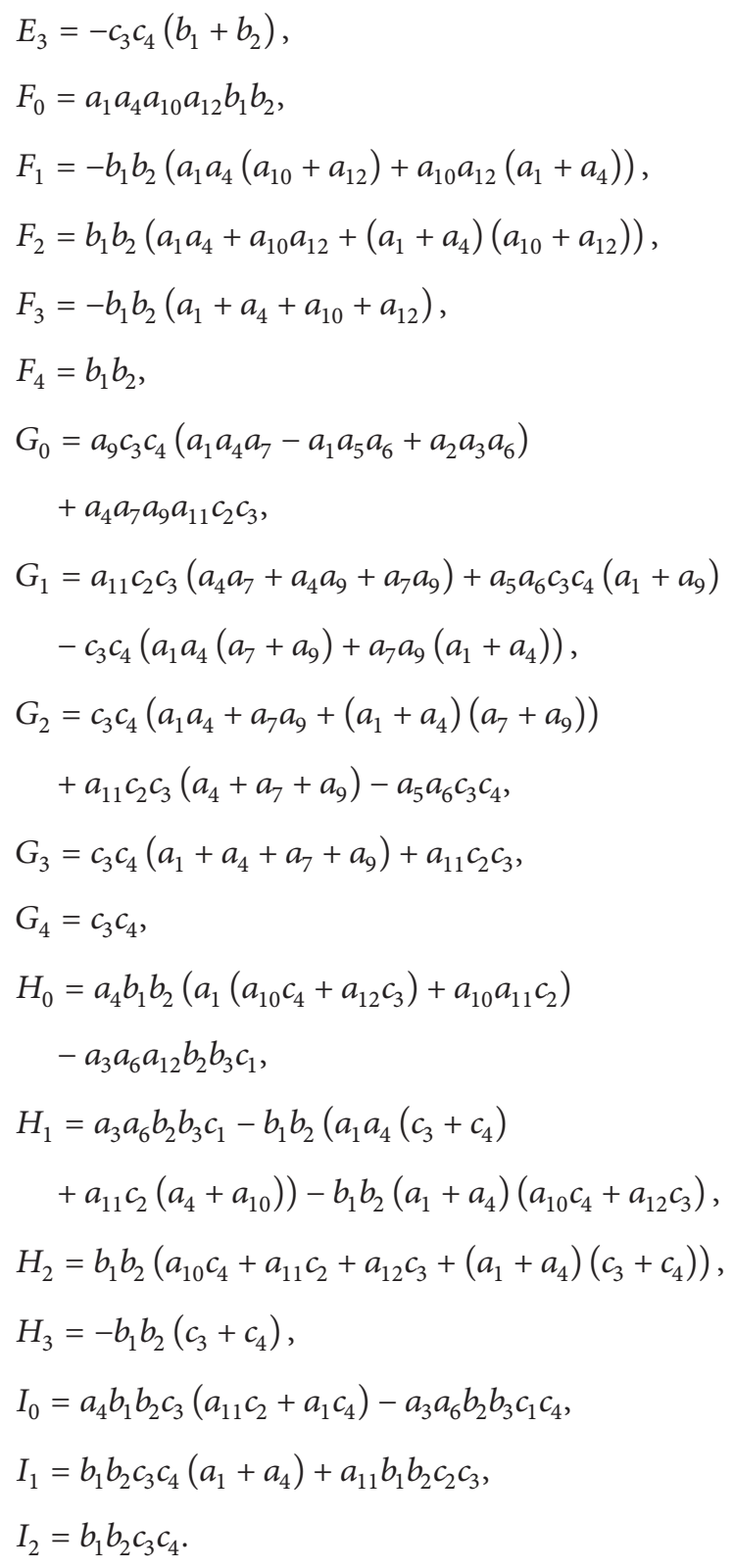

Case $1\left(\tau_{1}=\tau_{2}=0\right)$. When $\tau_{1}=\tau_{2}=0$, (6) becomes

$$
\begin{aligned}
\lambda^{6} & +A_{15} \lambda^{5}+A_{14} \lambda^{4}+A_{13} \lambda^{3}+A_{12} \lambda^{2}+A_{11} \lambda+A_{10} \\
& =0
\end{aligned}
$$

where

$$
\begin{aligned}
A_{10} & =A_{0}+B_{0}+C_{0}+D_{0}+E_{0}+F_{0}+G_{0}+H_{0}+I_{0}, \\
A_{11} & =A_{1}+B_{1}+C_{1}+D_{1}+E_{1}+F_{1}+G_{1}+H_{1}+I_{1}, \\
A_{12} & =A_{2}+B_{2}+C_{2}+D_{2}+E_{2}+F_{2}+G_{2}+H_{2}+I_{2}, \\
A_{13} & =A_{3}+B_{3}+C_{3}+D_{3}+E_{3}+F_{3}+G_{3}+H_{3}, \\
A_{14} & =A_{4}+B_{4}+C_{4}+D_{4}+E_{4}+F_{4}+G_{4}, A_{15} \\
& =A_{5}+B_{5}+C_{5} .
\end{aligned}
$$


Clearly, $D_{1}=A_{15}=\beta I_{*}+\rho+\gamma+\delta+\eta+\varepsilon+\theta+\chi+2 \alpha+6 d>$ 0 . Thus, if condition $\left(H_{1}\right)$ (see (10)) holds, then system (2) without delay is locally asymptotically stable:

$$
\begin{aligned}
D_{2} & =\operatorname{det}\left(\begin{array}{cc}
A_{15} & 1 \\
A_{13} & A_{14}
\end{array}\right)>0, \\
D_{3} & =\operatorname{det}\left(\begin{array}{ccc}
A_{15} & 1 & 0 \\
A_{13} & A_{14} & A_{15} \\
A_{11} & A_{12} & A_{13}
\end{array}\right)>0, \\
D_{4} & =\operatorname{det}\left(\begin{array}{cccc}
A_{05} & 1 & 0 & 0 \\
A_{03} & A_{14} & A_{15} & 1 \\
A_{01} & A_{12} & A_{13} & A_{14} \\
0 & A_{10} & A_{11} & A_{12}
\end{array}\right)>0, \\
D_{5} & =\operatorname{det}\left(\begin{array}{ccccc}
A_{15} & 1 & 0 & 0 & 0 \\
A_{13} & A_{14} & A_{15} & 1 & 0 \\
A_{11} & A_{12} & A_{13} & A_{14} & A_{15} \\
0 & A_{10} & A_{11} & A_{12} & A_{13} \\
0 & 0 & 0 & A_{10} & A_{11}
\end{array}\right)>0, \\
D_{6} & =A_{10}>0 .
\end{aligned}
$$

Case $2\left(\tau_{1}>0 ; \tau_{2}=0\right)$. Equation (6) equals

$$
\begin{gathered}
\lambda^{6}+A_{25} \lambda^{5}+A_{24} \lambda^{4}+A_{23} \lambda^{3}+A_{22} \lambda^{2}+A_{21} \lambda+A_{20} \\
+\left(B_{25} \lambda^{5}+B_{24} \lambda^{4}+B_{23} \lambda^{3}+B_{22} \lambda^{2}+B_{21} \lambda+B_{20}\right) \\
\cdot e^{-\lambda \tau_{1}}+\left(F_{24} \lambda^{4}+F_{23} \lambda^{3}+F_{22} \lambda^{2}+F_{21} \lambda+F_{20}\right) \\
\cdot e^{-2 \lambda \tau_{1}}=0
\end{gathered}
$$

where

$$
\begin{aligned}
& A_{20}=A_{0}+C_{0}+G_{0}, \\
& A_{21}=A_{1}+C_{1}+G_{1}, \\
& A_{22}=A_{2}+C_{2}+G_{2}, \\
& A_{23}=A_{3}+C_{3}+G_{3}, \\
& A_{24}=A_{4}+C_{4}+G_{4}, \\
& A_{25}=A_{5}+G_{5}, \\
& B_{20}=B_{0}+D_{0}+E_{0}, \\
& B_{21}=B_{1}+D_{1}+E_{1}, \\
& B_{22}=B_{2}+D_{2}+E_{2}, \\
& B_{23}=B_{3}+D_{3}+E_{3}, \\
& B_{24}=B_{4}+D_{4}+E_{4}, \\
& B_{25}=B_{5} .
\end{aligned}
$$

Multiplying $e^{\lambda \tau_{1}}$ on left and right of (11), one has

$$
\begin{aligned}
& B_{25} \lambda^{5}+B_{24} \lambda^{4}+B_{23} \lambda^{3}+B_{22} \lambda^{2}+B_{21} \lambda+B_{20}+\lambda^{6} \\
& +\left(A_{25} \lambda^{5}+A_{24} \lambda^{4}+A_{23} \lambda^{3}+A_{22} \lambda^{2}+A_{21} \lambda\right. \\
& \left.+A_{20}\right) e^{\lambda \tau_{1}}+\left(F_{24} \lambda^{4}+F_{23} \lambda^{3}+F_{22} \lambda^{2}+F_{21} \lambda\right. \\
& \left.+F_{20}\right) e^{-\lambda \tau_{1}}=0 .
\end{aligned}
$$

Assume that $\lambda=i \omega_{1}\left(\omega_{1}>0\right)$ is the root of $(13)$ :

$$
\begin{aligned}
& L_{21}\left(\omega_{1}\right) \cos \tau_{1} \omega_{1}-L_{22}\left(\omega_{1}\right) \sin \tau_{1} \omega_{1}=L_{23}\left(\omega_{1}\right), \\
& L_{24}\left(\omega_{1}\right) \sin \tau_{1} \omega_{1}+L_{25}\left(\omega_{1}\right) \cos \tau_{1} \omega_{1}=L_{26}\left(\omega_{1}\right),
\end{aligned}
$$

with

$$
\begin{aligned}
L_{21}\left(\omega_{1}\right)= & \left(A_{24}+F_{24}\right) \omega_{1}^{4}-\omega_{1}^{6}-\left(A_{22}+F_{22}\right) \omega_{1}^{2} \\
& +A_{20}+F_{20}, \\
L_{22}\left(\omega_{1}\right)= & A_{25} \omega_{1}^{5}-\left(A_{23}-F_{23}\right) \omega_{1}^{3}+\left(A_{21}-F_{21}\right) \omega_{1}, \\
L_{23}\left(\omega_{1}\right)= & B_{22} \omega_{1}^{2}-B_{24} \omega_{1}^{4}-B_{20}, \\
L_{24}\left(\omega_{1}\right)= & \left(A_{24}-F_{24}\right) \omega_{1}^{4}-\omega_{1}^{6}-\left(A_{22}-F_{22}\right) \omega_{1}^{2} \\
& +A_{20}-F_{20}, \\
L_{25}\left(\omega_{1}\right)= & A_{25} \omega_{1}^{5}-\left(A_{23}+F_{23}\right) \omega_{1}^{3}+\left(A_{21}+F_{21}\right) \omega_{1}, \\
L_{26}\left(\omega_{1}\right)= & B_{23} \omega_{1}^{3}-B_{25} \omega_{1}^{5}-B_{21} \omega_{1} .
\end{aligned}
$$

Thus, one can obtain the expressions of $\cos \tau_{1} \omega_{1}$ and $\sin \tau_{1} \omega_{1}$ as follows:

$$
\begin{aligned}
& \cos \tau_{1} \omega_{1} \\
& =\frac{L_{22}\left(\omega_{1}\right) \times L_{26}\left(\omega_{1}\right)+L_{23}\left(\omega_{1}\right) \times L_{24}\left(\omega_{1}\right)}{L_{21}\left(\omega_{1}\right) \times L_{24}\left(\omega_{1}\right)+L_{22}\left(\omega_{1}\right) \times L_{25}\left(\omega_{1}\right)},
\end{aligned}
$$

$\sin \tau_{1} \omega_{1}$

$$
=\frac{L_{21}\left(\omega_{1}\right) \times L_{26}\left(\omega_{1}\right)-L_{23}\left(\omega_{1}\right) \times L_{25}\left(\omega_{1}\right)}{L_{21}\left(\omega_{1}\right) \times L_{24}\left(\omega_{1}\right)+L_{22}\left(\omega_{1}\right) \times L_{25}\left(\omega_{1}\right)} .
$$

Then, we can get

$$
\cos ^{2} \tau_{1} \omega_{1}+\sin ^{2} \tau_{1} \omega_{1}=1 .
$$

Suppose that $\left(H_{21}\right)$ (see (17)) has at least one positive root.

If condition $\left(H_{21}\right)$ holds, then there exists $\omega_{10}>0$ such that (13) has a pair of purely imaginary roots $\pm i \omega_{10}$. For $\omega_{10}$,

$$
\begin{aligned}
\tau_{10} & =\frac{1}{\omega_{10}} \\
& \times \arccos \left\{\frac{L_{22}\left(\omega_{10}\right) \times L_{26}\left(\omega_{10}\right)+L_{23}\left(\omega_{10}\right) \times L_{24}\left(\omega_{10}\right)}{L_{21}\left(\omega_{10}\right) \times L_{24}\left(\omega_{10}\right)+L_{22}\left(\omega_{10}\right) \times L_{25}\left(\omega_{10}\right)}\right\} .
\end{aligned}
$$

Differentiating (13) with respect to $\tau_{1}$, one has

$$
\left[\frac{d \lambda}{d \tau_{1}}\right]^{-1}=\frac{F_{21}(\lambda)}{F_{22}(\lambda)}-\frac{\tau_{1}}{\lambda},
$$


where

$$
\begin{aligned}
& F_{21}(\lambda)=5 B_{25} \lambda^{4}+4 B_{24} \lambda^{4}+3 B_{23} \lambda^{2}+2 B_{22} \lambda+B_{21} \\
& +\left(6 \lambda^{5}+5 A_{25} \lambda^{4}+4 A_{24} \lambda^{3}+3 A_{23} \lambda^{2}+2 A_{22} \lambda\right. \\
& \left.+A_{21}\right) e^{\lambda \tau_{1}}+\left(4 F_{24} \lambda^{3}+3 F_{23} \lambda^{2}+2 F_{22} \lambda+F_{21}\right) \\
& \cdot e^{-\lambda \tau_{1}}, \\
& F_{22}(\lambda)=\left(4 F_{24} \lambda^{4}+3 F_{23} \lambda^{3}+2 F_{22} \lambda^{2}+F_{21} \lambda\right) e^{-\lambda \tau_{1}} \\
& \quad-\left(\lambda^{7}+A_{25} \lambda^{6}+A_{24} \lambda^{5}+A_{23} \lambda^{4}+A_{22} \lambda^{3}+A_{21} \lambda^{2}\right. \\
& \left.+A_{20} \lambda\right) e^{\lambda \tau_{1}} .
\end{aligned}
$$

Thus,

$$
\operatorname{Re}\left[\frac{d \lambda}{d \tau_{1}}\right]_{\lambda=i \omega_{10}}^{-1}=\frac{G_{2 R} \times H_{2 R}+G_{2 I} \times H_{2 I}}{H_{2 R}^{2}+H_{2 I}^{2}}
$$

with

$$
\begin{aligned}
G_{2 R} & =5 B_{25} \omega_{10}^{4}-3 B_{23} \omega_{10}^{2}+B_{21}+\left(5 A_{25} \omega_{10}^{4}\right. \\
& \left.-3\left(A_{23}+F_{23}\right) \omega_{10}^{2}+A_{21}+F_{21}\right) \cos \tau_{10} \omega_{10} \\
& -\left(6 \omega_{10}^{5}-4\left(A_{24}-F_{24}\right) \omega_{10}^{3}+2\left(A_{22}-F_{22}\right) \omega_{10}\right) \\
& \cdot \sin \tau_{10} \omega_{10}, \\
G_{2 I} & =2 B_{22} \omega_{10}-4 B_{24} \omega_{10}^{3}+\left(5 A_{25} \omega_{10}^{4}\right. \\
& \left.-3\left(A_{23}-F_{23}\right) \omega_{10}^{2}+A_{21}-F_{21}\right) \sin \tau_{10} \omega_{10} \\
& +\left(6 \omega_{10}^{5}-4\left(A_{24}+F_{24}\right) \omega_{10}^{3}+2\left(A_{22}+F_{22}\right) \omega_{10}\right) \\
& \cdot \cos \tau_{10} \omega_{10}, \\
H_{2 R} & =\left(\left(F_{21}+A_{20}\right) \omega_{10}+A_{24} \omega_{10}^{5}-\omega_{10}^{7}\right. \\
& \left.-\left(3 F_{23}+A_{22}\right) \omega_{10}^{3}\right) \sin \tau_{10} \omega_{10}+\left(\left(4 F_{24}-A_{23}\right) \omega_{10}^{4}\right. \\
& \left.-\left(2 F_{22}-A_{21}\right) \omega_{10}^{2}-A_{25} \omega_{10}^{6}\right) \cos \tau_{10} \omega_{10}, \\
& -\left(\left(4 F_{24}+A_{23}\right) \omega_{10}^{4}-\left(2 F_{22}-A_{21}\right) \omega_{10}^{2}\right. \\
H_{2 I} & =\left(\left(F_{21}-A_{20}\right) \omega_{10}-A_{24} \omega_{10}^{5}+\omega_{10}^{7}\right. \\
- & \left.\left(3 F_{23}-A_{22}\right) \omega_{10}^{3}\right) \cos \tau_{10} \omega_{10} . \\
& \\
& \\
&
\end{aligned}
$$

Thus, if condition $\left(H_{22}\right) G_{2 R} \times H_{2 R}+G_{2 I} \times H_{2 I} \neq 0$ holds, then $\operatorname{Re}\left[d \lambda / d \tau_{1}\right]_{\lambda=i \omega_{10}} \neq 0$. Based on the Hopf bifurcation theorem in [25], we have the following results.

Theorem 1. Suppose that conditions $\left(H_{1}\right),\left(H_{21}\right)$, and $\left(H_{22}\right)$ hold for system (2). The viral equilibrium $P_{*}\left(S_{*}, E_{*}, I_{*}\right.$, $\left.Q_{*}, R_{*}, V_{*}\right)$ is locally asymptotically stable when $\tau_{1} \in\left[0, \tau_{10}\right)$ and a Hopf bifurcation occurs at the viral equilibrium $P_{*}\left(S_{*}, E_{*}, I_{*}, Q_{*}, R_{*}, V_{*}\right)$ when $\tau_{1}=\tau_{10}$.
Case $3\left(\tau_{1}=0 ; \tau_{2}>0\right)$. Equation (6) becomes

$$
\begin{aligned}
\lambda^{6} & +A_{35} \lambda^{5}+A_{34} \lambda^{4}+A_{33} \lambda^{3}+A_{32} \lambda^{2}+A_{31} \lambda+A_{30} \\
& +\left(C_{35} \lambda^{5}+C_{34} \lambda^{4}+C_{33} \lambda^{3}+C_{32} \lambda^{2}+C_{31} \lambda+C_{30}\right) \\
& \cdot e^{-\lambda \tau_{2}}+\left(G_{34} \lambda^{4}+G_{33} \lambda^{3}+G_{32} \lambda^{2}+G_{31} \lambda+G_{30}\right) \\
& \cdot e^{-2 \lambda \tau_{2}}=0
\end{aligned}
$$

where

$$
\begin{aligned}
& A_{30}=A_{0}+B_{0}+F_{0}, \\
& A_{31}=A_{1}+B_{1}+F_{1}, \\
& A_{32}=A_{2}+B_{2}+F_{2}, \\
& A_{33}=A_{3}+B_{3}+F_{3}, \\
& A_{34}=A_{4}+B_{4}+F_{4}, \\
& A_{35}=A_{5}+B_{5}, \\
& C_{30}=C_{0}+D_{0}+H_{0}, \\
& C_{31}=C_{1}+D_{1}+H_{1}, \\
& C_{32}=C_{2}+D_{2}+H_{2}, \\
& C_{33}=C_{3}+D_{3}+H_{3}, \\
& C_{34}=C_{4}+D_{4}, \\
& C_{35}=C_{5}, \\
& G_{30}=E_{0}+G_{0}+I_{0}, \\
& G_{31}=E_{1}+G_{1}+I_{1}, \\
& G_{32}=E_{2}+G_{2}+I_{2}, \\
& G_{33}=E_{3}+G_{3}, \\
& G_{34}=G_{4} .
\end{aligned}
$$

Multiplying $e^{\lambda \tau_{2}}$ on left and right of (23), one has

$$
\begin{aligned}
& C_{35} \lambda^{5}+C_{34} \lambda^{4}+C_{33} \lambda^{3}+C_{32} \lambda^{2}+C_{31} \lambda+C_{30}+\left(\lambda^{6}\right. \\
& +A_{35} \lambda^{5}+A_{34} \lambda^{4}+A_{33} \lambda^{3}+A_{32} \lambda^{2}+A_{31} \lambda \\
& \left.+A_{30}\right) e^{\lambda \tau_{2}}+\left(G_{34} \lambda^{4}+G_{33} \lambda^{3}+G_{32} \lambda^{2}+G_{31} \lambda\right. \\
& \left.\quad+G_{30}\right) e^{-\lambda \tau_{2}}=0 .
\end{aligned}
$$

Let $\lambda=i \omega_{2}\left(\omega_{2}>0\right)$ be the root of (25):

$$
\begin{aligned}
& L_{31}\left(\omega_{2}\right) \cos \tau_{2} \omega_{2}-L_{32}\left(\omega_{2}\right) \sin \tau_{2} \omega_{2}=L_{33}\left(\omega_{2}\right), \\
& L_{34}\left(\omega_{2}\right) \sin \tau_{2} \omega_{2}+L_{35}\left(\omega_{2}\right) \cos \tau_{2} \omega_{2}=L_{36}\left(\omega_{2}\right),
\end{aligned}
$$


with

$$
\begin{aligned}
L_{31}\left(\omega_{2}\right)= & \left(A_{34}+G_{34}\right) \omega_{2}^{4}-\omega_{2}^{6}-\left(A_{32}+G_{32}\right) \omega_{2}^{2} \\
& +A_{30}+G_{30}, \\
L_{32}\left(\omega_{2}\right)= & A_{35} \omega_{2}^{5}-\left(A_{33}-G_{33}\right) \omega_{2}^{3} \\
& +\left(A_{31}-G_{31}\right) \omega_{2}, \\
L_{33}\left(\omega_{2}\right)= & C_{32} \omega_{2}^{2}-C_{34} \omega_{2}^{4}-C_{30}, \\
L_{34}\left(\omega_{2}\right)= & \left(A_{34}-G_{34}\right) \omega_{2}^{4}-\omega_{2}^{6}-\left(A_{32}-G_{32}\right) \omega_{2}^{2} \\
& +A_{30}-G_{30}, \\
L_{35}\left(\omega_{2}\right)= & A_{35} \omega_{2}^{5}-\left(A_{33}+G_{33}\right) \omega_{2}^{3} \\
& +\left(A_{31}+G_{31}\right) \omega_{2}, \\
L_{36}\left(\omega_{2}\right)= & C_{33} \omega_{2}^{3}-C_{35} \omega_{2}^{5}-C_{31} \omega_{2} .
\end{aligned}
$$

Then,

$\cos \tau_{2} \omega_{2}$

$$
=\frac{L_{32}\left(\omega_{2}\right) \times L_{36}\left(\omega_{2}\right)+L_{33}\left(\omega_{2}\right) \times L_{34}\left(\omega_{2}\right)}{L_{31}\left(\omega_{2}\right) \times L_{34}\left(\omega_{2}\right)+L_{32}\left(\omega_{2}\right) \times L_{35}\left(\omega_{2}\right)},
$$

$\sin \tau_{2} \omega_{2}$

$$
=\frac{L_{31}\left(\omega_{2}\right) \times L_{36}\left(\omega_{2}\right)-L_{33}\left(\omega_{2}\right) \times L_{35}\left(\omega_{2}\right)}{L_{31}\left(\omega_{2}\right) \times L_{34}\left(\omega_{2}\right)+L_{32}\left(\omega_{2}\right) \times L_{35}\left(\omega_{2}\right)} .
$$

And the equation following equation regarding $\tau_{2}$ can be obtained:

$$
\cos ^{2} \tau_{2} \omega_{2}+\sin ^{2} \tau_{2} \omega_{2}=1
$$

Suppose that $\left(H_{31}\right)$ (see (29)) has at least one positive root.

If condition $\left(H_{31}\right)$ holds, then there exists $\omega_{20}>0$ such that (25) has a pair of purely imaginary roots $\pm i \omega_{20}$. For $\omega_{20}$,

$$
\begin{aligned}
\tau_{20} & =\frac{1}{\omega_{20}} \\
& \times \arccos \left\{\frac{L_{32}\left(\omega_{20}\right) \times L_{36}\left(\omega_{20}\right)+L_{33}\left(\omega_{20}\right) \times L_{34}\left(\omega_{20}\right)}{L_{31}\left(\omega_{20}\right) \times L_{34}\left(\omega_{20}\right)+L_{32}\left(\omega_{20}\right) \times L_{35}\left(\omega_{20}\right)}\right\} .
\end{aligned}
$$

Differentiate both sides of (25) with respect to $\tau_{2}$. Then,

$$
\left[\frac{d \lambda}{d \tau_{2}}\right]^{-1}=\frac{F_{31}(\lambda)}{F_{32}(\lambda)}-\frac{\tau_{2}}{\lambda},
$$

where

$$
\begin{aligned}
& F_{31}(\lambda)=5 C_{35} \lambda^{4}+4 C_{34} \lambda^{4}+3 C_{33} \lambda^{2}+2 C_{32} \lambda+C_{31} \\
& +\left(6 \lambda^{5}+5 A_{35} \lambda^{4}+4 A_{34} \lambda^{3}+3 A_{33} \lambda^{2}+2 A_{32} \lambda\right. \\
& \left.+A_{31}\right) e^{\lambda \tau_{2}}+\left(4 G_{34} \lambda^{3}+3 G_{33} \lambda^{2}+2 G_{32} \lambda+G_{31}\right) \\
& \quad \cdot e^{-\lambda \tau_{2}}, \\
& F_{32}(\lambda)=\left(4 G_{34} \lambda^{4}+3 G_{33} \lambda^{3}+2 G_{32} \lambda^{2}+G_{31} \lambda\right) e^{-\lambda \tau_{2}} \\
& \quad-\left(\lambda^{7}+A_{35} \lambda^{6}+A_{34} \lambda^{5}+A_{33} \lambda^{4}+A_{32} \lambda^{3}+A_{31} \lambda^{2}\right. \\
& \left.+A_{30} \lambda\right) e^{\lambda \tau_{2}} .
\end{aligned}
$$

Thus,

$$
\operatorname{Re}\left[\frac{d \lambda}{d \tau_{2}}\right]_{\lambda=i \omega_{20}}^{-1}=\frac{G_{3 R} \times H_{3 R}+G_{3 I} \times H_{3 I}}{H_{3 R}^{2}+H_{3 I}^{2}},
$$

with

$$
\begin{aligned}
G_{3 R} & =5 C_{35} \omega_{20}^{4}-C_{33} \omega_{20}^{2}+C_{31}+\left(5 A_{35} \omega_{20}^{4}\right. \\
- & \left.3\left(A_{33}+G_{33}\right) \omega_{20}^{2}+A_{31}+G_{31}\right) \cos \tau_{20} \omega_{20} \\
- & \left(6 \omega_{20}^{5}-4\left(A_{34}+G_{34}\right) \omega_{20}^{3}+2\left(A_{32}+G_{32}\right) \omega_{20}\right) \\
& \cdot \sin \tau_{20} \omega_{20}, \\
G_{3 I} & =2 C_{22} \omega_{20}-4 C_{24} \omega_{20}^{3}+\left(5 A_{35} \omega_{20}^{4}\right. \\
& \left.-3\left(A_{33}+G_{33}\right) \omega_{20}^{2}+A_{31}-G_{31}\right) \sin \tau_{20} \omega_{20} \\
& +\left(6 \omega_{20}^{5}-4\left(A_{34}+G_{34}\right) \omega_{20}^{3}+2\left(A_{32}+G_{32}\right) \omega_{20}\right) \\
& \cdot \cos \tau_{20} \omega_{20}, \\
H_{3 R} & =\left(\left(G_{31}+A_{30}\right) \omega_{20}+A_{34} \omega_{20}^{5}-\omega_{20}^{7}\right. \\
& \left.-\left(3 G_{33}+A_{32}\right) \omega_{20}^{3}\right) \sin \tau_{20} \omega_{20} \\
& +\left(\left(4 G_{34}-A_{33}\right) \omega_{20}^{4}-\left(2 G_{32}-A_{31}\right) \omega_{20}^{2}\right. \\
& \left.-A_{35} \omega_{20}^{6}\right) \cos \tau_{20} \omega_{20}, \\
H_{3 I} & =\left(\left(G_{31}-A_{30}\right) \omega_{20}-A_{34} \omega_{20}^{5}+\omega_{20}^{7}\right. \\
& \left.-\left(3 G_{33}-A_{32}\right) \omega_{20}^{3}\right) \cos \tau_{20} \omega_{20} \\
& +\left(\left(4 G_{34}+A_{33}\right) \omega_{20}^{4}-\left(2 G_{32}+A_{31}\right) \omega_{20}^{2}\right. \\
& \left.-A_{35} \omega_{20}^{6}\right) \sin \tau_{20} \omega_{20} \cdot
\end{aligned}
$$

Similar to Case 2, we know that if condition $\left(H_{32}\right) G_{3 R} \times$ $H_{3 R}+G_{3 I} \times H_{3 I} \neq 0$ holds, then $\operatorname{Re}\left[d \lambda / d \tau_{2}\right]_{\lambda=i \omega_{20}} \neq 0$. In conclusion, we have the following results.

Theorem 2. Suppose that conditions $\left(H_{1}\right),\left(H_{31}\right)$, and $\left(H_{32}\right)$ hold for system (2). The viral equilibrium $P_{*}\left(S_{*}, E_{*}, I_{*}\right.$, 
$\left.Q_{*}, R_{*}, V_{*}\right)$ is locally asymptotically stable when $\tau_{2} \in\left[0, \tau_{20}\right)$ and a Hopf bifurcation occurs at the viral equilibrium $P_{*}\left(S_{*}, E_{*}, I_{*}, Q_{*}, R_{*}, V_{*}\right)$ when $\tau_{2}=\tau_{20}$.

Case $4\left(\tau_{1}>0 ; \tau_{2} \in\left(0, \tau_{20}\right)\right)$. Regarding $\tau_{1}$ as the bifurcation parameter when $\tau_{2} \in\left(0, \tau_{20}\right)$, multiplying by $e^{\lambda \tau_{1}}$, (6) becomes

$$
\begin{aligned}
& B_{5} \lambda^{5}+B_{4} \lambda^{4}+B_{3} \lambda^{3}+B_{2} \lambda^{2}+B_{1} \lambda+B_{0}+\left(D_{4} \lambda^{4}\right. \\
& \left.+D_{3} \lambda^{3}+D_{2} \lambda^{2}+D_{1} \lambda+D_{0}\right) e^{-\lambda \tau_{2}}+\left(E_{3} \lambda^{3}+E_{2} \lambda^{2}\right. \\
& \left.+E_{1} \lambda+E_{0}\right) e^{-2 \lambda \tau_{2}}+\left(\lambda^{6}+A_{5} \lambda^{5}+A_{4} \lambda^{4}+A_{3} \lambda^{3}\right. \\
& \left.+A_{2} \lambda^{2}+A_{1} \lambda+A_{0}\right) e^{\lambda \tau_{1}}+\left(C_{5} \lambda^{5}+C_{4} \lambda^{4}+C_{3} \lambda^{3}\right. \\
& \left.+C_{2} \lambda^{2}+C_{1} \lambda+C_{0}\right) e^{\lambda\left(\tau_{1}-\tau_{2}\right)}+\left(F_{4} \lambda^{4}+F_{3} \lambda^{3}\right. \\
& \left.+F_{2} \lambda^{2}+F_{1} \lambda+F_{0}\right) e^{-\lambda \tau_{1}}+\left(G_{4} \lambda^{4}+G_{3} \lambda^{3}+G_{2} \lambda^{2}\right. \\
& \left.+G_{1} \lambda+G_{0}\right) e^{\lambda\left(\tau_{1}-2 \tau_{2}\right)}+\left(H_{3} \lambda^{3}+H_{2} \lambda^{2}+H_{1} \lambda\right. \\
& \left.+H_{0}\right) e^{-\lambda\left(\tau_{1}+\tau_{2}\right)}+\left(I_{2} \lambda^{2}+I_{1} \lambda+I_{0}\right) e^{-\lambda\left(\tau_{1}+2 \tau_{2}\right)} .
\end{aligned}
$$

Let $\lambda=i \omega_{1}^{*}\left(\omega_{1}^{*}>0\right)$ be the root of (35), and for the convenience we still denote $\omega_{1}^{*}$ as $\omega_{1}$; then,

$$
\begin{aligned}
& L_{41}\left(\omega_{1}\right) \cos \tau_{1} \omega_{1}-L_{42}\left(\omega_{1}\right) \sin \tau_{1} \omega_{1}=L_{43}\left(\omega_{1}\right), \\
& L_{44}\left(\omega_{1}\right) \sin \tau_{1} \omega_{1}+L_{45}\left(\omega_{1}\right) \cos \tau_{1} \omega_{1}=L_{46}\left(\omega_{1}\right),
\end{aligned}
$$

where

$$
\begin{aligned}
& L_{41}\left(\omega_{1}\right)=\left(A_{4}+F_{4}\right) \omega_{1}^{4}-\omega_{1}^{6}-\left(A_{2}+F_{2}\right) \omega_{1}^{2}+A_{0} \\
& +F_{0}+\left(C_{4} \omega_{1}^{4}-\left(C_{2}+H_{2}\right) \omega_{1}^{2}+C_{0}+H_{0}\right) \cos \tau_{2} \omega_{1} \\
& +\left(C_{5} \omega_{1}^{5}-\left(C_{3}+H_{3}\right) \omega_{1}^{3}+\left(C_{1}+H_{1}\right) \omega_{1}\right) \sin \tau_{2} \omega_{1} \\
& +\left(G_{4} \omega_{1}^{4}-\left(G_{2}+I_{2}\right) \omega_{1}^{2}+G_{0}+I_{0}\right) \cos 2 \tau_{2} \omega_{1} \\
& +\left(\left(G_{1}+I_{1}\right) \omega_{1}-G_{3} \omega_{1}^{3}\right) \sin 2 \tau_{2} \omega_{1}, \\
& L_{42}\left(\omega_{1}\right)=A_{5} \omega_{1}^{5}-\left(A_{3}-F_{3}\right) \omega_{1}^{3}+\left(A_{1}-F_{1}\right) \omega_{1} \\
& \quad+\left(C_{5} \omega_{1}^{5}-\left(C_{3}-H_{3}\right) \omega_{1}^{3}+\left(C_{1}-H_{1}\right) \omega_{1}\right) \cos \tau_{2} \omega_{1} \\
& \quad-\left(C_{4} \omega_{1}^{4}-\left(C_{2}-H_{2}\right) \omega_{1}^{2}+C_{0}-H_{0}\right) \sin \tau_{2} \omega_{1} \\
& \quad+\left(\left(G_{1}-I_{1}\right) \omega_{1}-G_{3} \omega_{1}^{3}\right) \cos 2 \tau_{2} \omega_{1} \\
& \quad-\left(G_{4} \omega_{1}^{4}-\left(G_{2}-I_{2}\right) \omega_{1}^{2}+G_{0}-I_{0}\right) \sin 2 \tau_{2} \omega_{1},
\end{aligned}
$$

$$
\begin{aligned}
L_{43} & \left(\omega_{1}\right)=B_{2} \omega_{1}^{2}-B_{4} \omega_{1}^{4}-B_{0} \\
& +\left(D_{3} \omega_{1}^{3}-D_{1} \omega_{1}\right) \sin \tau_{2} \omega_{1} \\
& +\left(D_{2} \omega_{1}^{2}-D_{4} \omega_{1}^{4}-D_{0}\right) \cos \tau_{2} \omega_{1} \\
& +\left(E_{3} \omega_{1}^{3}-E_{1} \omega_{1}\right) \sin \tau_{2} \omega_{1}+\left(E_{2} \omega_{1}^{2}-E_{0}\right) \cos \tau_{2} \omega_{1}, \\
L_{44} & \left(\omega_{1}\right)=\left(A_{4}-F_{4}\right) \omega_{1}^{4}-\omega_{1}^{6}-\left(A_{2}-F_{2}\right) \omega_{1}^{2}+A_{0} \\
& -F_{0}+\left(C_{4} \omega_{1}^{4}-\left(C_{2}-H_{2}\right) \omega_{1}^{2}+C_{0}-H_{0}\right) \cos \tau_{2} \omega_{1} \\
& +\left(C_{5} \omega_{1}^{5}-\left(C_{3}-H_{3}\right) \omega_{1}^{3}+\left(C_{1}-H_{1}\right) \omega_{1}\right) \sin \tau_{2} \omega_{1} \\
& +\left(G_{4} \omega_{1}^{4}-\left(G_{2}-I_{2}\right) \omega_{1}^{2}+G_{0}-I_{0}\right) \cos 2 \tau_{2} \omega_{1} \\
& +\left(\left(G_{1}-I_{1}\right) \omega_{1}-G_{3} \omega_{1}^{3}\right) \sin 2 \tau_{2} \omega_{1}, \\
L_{45} & \left(\omega_{1}\right)=A_{5} \omega_{1}^{5}-\left(A_{3}+F_{3}\right) \omega_{1}^{3}+\left(A_{1}+F_{1}\right) \omega_{1} \\
& +\left(C_{5} \omega_{1}^{5}-\left(C_{3}+H_{3}\right) \omega_{1}^{3}+\left(C_{1}+H_{1}\right) \omega_{1}\right) \cos \tau_{2} \omega_{1} \\
& -\left(C_{4} \omega_{1}^{4}-\left(C_{2}+H_{2}\right) \omega_{1}^{2}+C_{0}+H_{0}\right) \sin \tau_{2} \omega_{1} \\
& +\left(\left(G_{1}+I_{1}\right) \omega_{1}-G_{3} \omega_{1}^{3}\right) \cos 2 \tau_{2} \omega_{1} \\
& +\left(G_{4} \omega_{1}^{4}-\left(G_{2}+I_{2}\right) \omega_{1}^{2}+G_{0}+I_{0}\right) \sin 2 \tau_{2} \omega_{1}, \\
& +\left(D_{3} \omega_{1}^{3}-E_{1} \omega_{1}\right) \cos \tau_{2} \omega_{1}-\left(E_{2} \omega_{1}^{2}-E_{0}\right) \sin \tau_{2} \omega_{1} . \\
L_{46} & \left.\left(\omega_{1}\right)=B_{3} \omega_{1}^{3}-B_{3} \omega_{1}^{3}-B_{1} \omega_{1}\right) \cos \tau_{2} \omega_{1} \\
&
\end{aligned}
$$

Thus,

$\cos \tau_{1} \omega_{1}$

$$
=\frac{L_{42}\left(\omega_{1}\right) \times L_{46}\left(\omega_{1}\right)+L_{43}\left(\omega_{1}\right) \times L_{44}\left(\omega_{1}\right)}{L_{41}\left(\omega_{1}\right) \times L_{44}\left(\omega_{1}\right)+L_{42}\left(\omega_{1}\right) \times L_{45}\left(\omega_{1}\right)},
$$

$\sin \tau_{1} \omega_{1}$

$$
=\frac{L_{41}\left(\omega_{1}\right) \times L_{46}\left(\omega_{1}\right)-L_{43}\left(\omega_{1}\right) \times L_{45}\left(\omega_{1}\right)}{L_{41}\left(\omega_{1}\right) \times L_{44}\left(\omega_{1}\right)+L_{42}\left(\omega_{1}\right) \times L_{45}\left(\omega_{1}\right)} .
$$

Then, we get

$$
\cos ^{2} \tau_{1} \omega_{1}+\sin ^{2} \tau_{1} \omega_{1}=1 .
$$

Suppose that $\left(H_{41}\right)$ (see (39)) has at least one positive root.

If $\left(H_{41}\right)$ holds, then there exists $\omega_{10}>0$ such that (35) has a pair of purely imaginary roots $\pm i \omega_{10}^{*}$. For $\omega_{10}^{*}$,

$$
\begin{aligned}
\tau_{10}^{*} & =\frac{1}{\omega_{10}^{*}} \\
& \times \arccos \left\{\frac{L_{42}\left(\omega_{10}^{*}\right) \times L_{46}\left(\omega_{10}^{*}\right)+L_{43}\left(\omega_{10}^{*}\right) \times L_{44}\left(\omega_{10}^{*}\right)}{L_{41}\left(\omega_{10}^{*}\right) \times L_{44}\left(\omega_{10}^{*}\right)+L_{42}\left(\omega_{10}^{*}\right) \times L_{45}\left(\omega_{10}^{*}\right)}\right\} .
\end{aligned}
$$


Differentiating both sides of (25) with respect to $\tau_{2}$,

$$
\left[\frac{d \lambda}{d \tau_{1}}\right]^{-1}=\frac{F_{41}(\lambda)}{F_{42}(\lambda)}-\frac{\tau_{1}}{\lambda}
$$

where

$$
\begin{aligned}
F_{41} & (\lambda)=5 B_{5} \lambda^{4}+4 B_{4} \lambda^{3}+3 B_{3} \lambda^{2}+2 B_{2} \lambda+B_{1} \\
& +\left(\left(4 D_{4}-\tau_{2} D_{3}\right) \lambda^{3}-\tau_{2} D_{4} \lambda^{4}+\left(3 D_{3}-\tau_{2} D_{2}\right) \lambda^{2}\right. \\
& \left.+\left(2 D_{2}-D_{1}\right) \lambda+D_{1}-\tau_{2} D_{0}\right) e^{-\lambda \tau_{2}}+\left(6 \lambda^{5}\right. \\
& \left.+5 A_{5} \lambda^{4}+4 A_{4} \lambda^{3}+3 A_{3} \lambda^{2}+2 A_{2} \lambda+A_{1}\right) e^{\lambda \tau_{1}} \\
& +\left(\left(3 E_{3}-2 \tau_{2} E_{2}\right) \lambda^{2}-2 \tau_{2} E_{3} \lambda^{3}+2\left(E_{2}-\tau_{2} E_{1}\right) \lambda\right. \\
& \left.+E_{1}-2 \tau_{2} E_{0}\right) e^{-2 \lambda \tau_{2}}+\left(\left(5 C_{5}-\tau_{2} C_{4}\right) \lambda^{4}-\tau_{2} C_{5} \lambda^{5}\right. \\
& +\left(4 C_{4}-\tau_{2} C_{3}\right) \lambda^{3}+\left(3 C_{3}-\tau_{2} C_{2}\right) \lambda^{2} \\
& \left.+\left(2 C_{2}-\tau_{2} C_{1}\right) \lambda+C_{1}-\tau_{2} C_{0}\right) e^{\lambda\left(\tau_{1}-\tau_{2}\right)}+\left(4 F_{4} \lambda^{3}\right. \\
& \left.+3 F_{3} \lambda^{2}+2 F_{2} \lambda+F_{1}\right) e^{-\lambda \tau_{1}}+\left(\left(3 H_{3}-\tau_{2} H_{2}\right) \lambda^{2}\right. \\
& \left.+C_{4} \lambda^{5}+C_{3} \lambda^{4}+C_{2} \lambda^{3}+C_{1} \lambda^{2}+C_{0} \lambda\right) e^{\lambda\left(\tau_{1}-\tau_{2}\right)} \\
& -\left(G_{4} \lambda^{5}+G_{3} \lambda^{4}+G_{2} \lambda^{3}+\left(2 H_{2}-\tau_{2} H_{1}\right) \lambda+H^{2}+G_{0} \lambda\right) e^{\lambda\left(\tau_{1}-2 \tau_{2}\right)} \\
& \left.+\tau_{2} H_{0}\right) \\
& +e^{-\lambda\left(\tau_{1}+\tau_{2}\right)}+\left(\left(4 G_{4}-2 \tau_{2} G_{3}\right) \lambda^{3}-2 \tau_{2} G_{4} \lambda^{4}\right. \\
& +\left(3 G_{3}-\tau_{2} G_{2}\right) \lambda^{2}+2\left(G_{2}-\tau_{2} G_{1}\right) \lambda+G_{1} \lambda^{3}+\lambda\left(\tau_{1}+2 \tau_{2}\right)-\left(\lambda^{7}+A_{5} \lambda^{6}+A_{4} \lambda^{5}\right. \\
& \left.-2 \tau_{2} G_{0}\right) e^{\lambda\left(\tau_{1}-2 \tau_{2}\right)}+\left(2\left(I_{2}-\tau_{2} I_{1}\right) \lambda-2 \tau_{2} I_{2} \lambda^{2}+I_{1}\right. \\
& \left.-2 I_{0}\right) e^{-\lambda\left(\tau_{1}+2 \tau_{2}\right)},
\end{aligned}
$$

Define

$$
\operatorname{Re}\left[\frac{d \lambda}{d \tau_{1}}\right]_{\lambda=i \omega_{10}^{*}}^{-1}=\frac{G_{4 R} \times H_{4 R}+G_{4 I} \times H_{4 I}}{H_{4 R}^{2}+H_{4 I}^{2}} .
$$

Similar to Case 2, we know that if condition $\left(H_{42}\right) G_{4 R} \times$ $H_{4 R}+G_{4 I} \times H_{4 I} \neq 0$ holds, then $\operatorname{Re}\left[d \lambda / d \tau_{1}\right]_{\lambda=i \omega_{10}^{*}} \neq 0$. Thus, we have the following results.

Theorem 3. Let $\tau_{2} \in\left(0, \tau_{20}\right)$ and suppose that conditions $\left(H_{1}\right)$, $\left(H_{41}\right)$, and $\left(H_{42}\right)$ hold for system (2). The viral equilibrium $P_{*}\left(S_{*}, E_{*}, I_{*}, Q_{*}, R_{*}, V_{*}\right)$ is locally asymptotically stable when $\tau_{1} \in\left[0, \tau_{10}^{*}\right)$ and a Hopf bifurcation occurs at the viral equilibrium $P_{*}\left(S_{*}, E_{*}, I_{*}, Q_{*}, R_{*}, V_{*}\right)$ when $\tau_{1}=\tau_{10}^{*}$.
Case $5\left(\tau_{1} \in\left(0, \tau_{10}\right) ; \tau_{2}>0\right)$. Regarding $\tau_{2}$ as the bifurcation parameter when $\tau_{1} \in\left(0, \tau_{10}\right)$, multiplying by $e^{\lambda \tau_{2}}$, (6) becomes

$$
\begin{aligned}
& C_{5} \lambda^{5}+C_{4} \lambda^{4}+C_{3} \lambda^{3}+C_{2} \lambda^{2}+C_{1} \lambda+C_{0}+\left(D_{4} \lambda^{4}\right. \\
& \left.+D_{3} \lambda^{3}+D_{2} \lambda^{2}+D_{1} \lambda+D_{0}\right) e^{-\lambda \tau_{1}}+\left(H_{3} \lambda^{3}\right. \\
& \left.+H_{2} \lambda^{2}+H_{1} \lambda+H_{0}\right) e^{-2 \lambda \tau_{1}}+\left(G_{4} \lambda^{4}+G_{3} \lambda^{3}\right. \\
& \left.+G_{2} \lambda^{2}+G_{1} \lambda+G_{0}\right) e^{-\lambda \tau_{2}}+\left(\lambda^{6}+A_{5} \lambda^{5}+A_{4} \lambda^{4}\right. \\
& \left.+A_{3} \lambda^{3}+A_{2} \lambda^{2}+A_{1} \lambda+A_{0}\right) e^{\lambda \tau_{2}}+\left(B_{5} \lambda^{5}+B_{4} \lambda^{4}\right. \\
& \left.+B_{3} \lambda^{3}+B_{2} \lambda^{2}+B_{1} \lambda+B_{0}\right) e^{\lambda\left(\tau_{2}-\tau_{1}\right)}+\left(E_{3} \lambda^{3}\right. \\
& \left.+E_{2} \lambda^{2}+E_{1} \lambda+E_{0}\right) e^{-\lambda\left(\tau_{2}+\tau_{1}\right)}+\left(F_{4} \lambda^{4}+F_{3} \lambda^{3}\right. \\
& \left.+F_{2} \lambda^{2}+F_{1} \lambda+F_{0}\right) e^{\lambda\left(\tau_{2}-2 \tau_{1}\right)}+\left(I_{2} \lambda^{2}+I_{1} \lambda+I_{0}\right) \\
& +e^{-\lambda\left(\tau_{2}+2 \tau_{1}\right)}
\end{aligned}
$$

Let $\lambda=i \omega_{2}^{*}\left(\omega_{2}^{*}>0\right)$ be the root of (44), and for the convenience we still denote $\omega_{2}^{*}$ as $\omega_{2}$; then,

$$
\begin{aligned}
& L_{51}\left(\omega_{2}\right) \cos \tau_{2} \omega_{2}-L_{52}\left(\omega_{1}\right) \sin \tau_{2} \omega_{2}=L_{53}\left(\omega_{2}\right), \\
& L_{54}\left(\omega_{2}\right) \sin \tau_{2} \omega_{2}+L_{55}\left(\omega_{1}\right) \cos \tau_{2} \omega_{2}=L_{56}\left(\omega_{2}\right),
\end{aligned}
$$

where

$$
\begin{aligned}
L_{51} & \left(\omega_{2}\right) \\
= & \left(A_{4}+G_{4}\right) \omega_{2}^{4}-\omega_{2}^{6}-\left(A_{2}+G_{2}\right) \omega_{2}^{2}+A_{0}+G_{0} \\
& +\left(B_{4} \omega_{2}^{4}-\left(B_{2}+E_{2}\right) \omega_{2}^{2}+B_{0}+E_{0}\right) \cos \tau_{1} \omega_{2} \\
& +\left(B_{5} \omega_{2}^{5}-\left(B_{3}+E_{3}\right) \omega_{2}^{3}+\left(B_{1}-E_{1}\right) \omega_{2}\right) \sin \tau_{1} \omega_{2} \\
& +\left(F_{4} \omega_{2}^{4}-\left(F_{2}+I_{2}\right) \omega_{2}^{2}+F_{0}+I_{0}\right) \cos 2 \tau_{1} \omega_{2} \\
& +\left(\left(F_{1}+I_{1}\right) \omega_{2}-F_{3} \omega_{2}^{3}\right) \sin 2 \tau_{1} \omega_{2}, \\
L_{52} & \left(\omega_{2}\right) \\
= & A_{5} \omega_{2}^{5}-\left(A_{3}-G_{3}\right) \omega_{2}^{3}+\left(A_{1}-G_{1}\right) \omega_{2} \\
& +\left(\left(B_{2}-E_{2}\right) \omega_{2}^{2}-B_{4} \omega_{2}^{4}+E_{0}-B_{0}\right) \sin \tau_{1} \omega_{2} \\
& +\left(B_{5} \omega_{2}^{5}-\left(B_{3}-E_{3}\right) \omega_{2}^{3}+\left(B_{1}-E_{1}\right) \omega_{2}\right) \cos \tau_{1} \omega_{2} \\
& -\left(F_{4} \omega_{2}^{4}-\left(F_{2}-I_{2}\right) \omega_{2}^{2}+F_{0}-I_{0}\right) \sin 2 \tau_{1} \omega_{2} \\
& +\left(\left(F_{1}-I_{1}\right) \omega_{2}-F_{3} \omega_{2}^{3}\right) \cos 2 \tau_{1} \omega_{2},
\end{aligned}
$$




$$
\begin{aligned}
L_{53} & \left(\omega_{2}\right) \\
= & C_{2} \omega_{2}^{2}-C_{4} \omega_{2}^{4}-C_{0}+\left(D_{3} \omega_{2}^{3}-D_{1} \omega_{2}\right) \sin \tau_{1} \omega_{2} \\
& +\left(D_{2} \omega_{2}^{2}-D_{4} \omega_{2}^{4}-D_{0}\right) \cos \tau_{1} \omega_{2} \\
& +\left(H_{3} \omega_{2}^{3}-H_{1} \omega_{2}\right) \sin 2 \tau_{1} \omega_{2} \\
& +\left(H_{2} \omega_{2}^{2}-H_{0}\right) \cos 2 \tau_{1} \omega_{2}, \\
L_{54} & \left(\omega_{2}\right) \\
= & \left(A_{4}-G_{4}\right) \omega_{2}^{4}-\omega_{2}^{6}-\left(A_{2}-G_{2}\right) \omega_{2}^{2}+A_{0}-G_{0} \\
& +\left(B_{4} \omega_{2}^{4}-\left(B_{2}-E_{2}\right) \omega_{2}^{2}+B_{0}-E_{0}\right) \cos \tau_{1} \omega_{2} \\
& +\left(B_{5} \omega_{2}^{5}-\left(B_{3}-E_{3}\right) \omega_{2}^{3}+\left(B_{1}-E_{1}\right) \omega_{2}\right) \sin \tau_{1} \omega_{2} \\
& +\left(F_{4} \omega_{2}^{4}-\left(F_{2}-I_{2}\right) \omega_{2}^{2}+F_{0}-I_{0}\right) \cos 2 \tau_{1} \omega_{2} \\
& +\left(\left(F_{1}-I_{1}\right) \omega_{2}-F_{3} \omega_{2}^{3}\right) \sin 2 \tau_{1} \omega_{2}, \\
L_{55} & \left(\omega_{2}\right) \\
= & A_{5} \omega_{2}^{5}-\left(A_{3}+G_{3}\right) \omega_{2}^{3}+\left(A_{1}+G_{1}\right) \omega_{2} \\
& -\left(B_{4} \omega_{2}^{4}-\left(B_{2}+E_{2}\right) \omega_{2}^{2}+E_{0}+B_{0}\right) \sin \tau_{1} \omega_{2} \\
& +\left(H_{3} \omega_{2}^{3}-H_{1} \omega_{2}\right) \cos 2 \tau_{1} \omega_{2} \\
& +\left(B_{5} \omega_{2}^{5}-\left(B_{3}+E_{3}\right) \omega_{2}^{3}+\left(B_{1}+E_{1}\right) \omega_{2}\right) \cos \tau_{1} \omega_{2} \omega_{2} . \\
& -\left(F_{4} \omega_{2}^{4}-\left(F_{2}+I_{2}\right) \omega_{2}^{2}+F_{0}-I_{0}\right) \sin 2 \tau_{1} \omega_{2} \\
& +\left(\left(F_{1}+I_{1}\right) \omega_{2}-F_{3} \omega_{2}^{3}\right) \cos 2 \tau_{1} \omega_{2}, \\
L_{56} & \left(\omega_{2}\right) \\
= & C_{3} \omega_{2}^{3}-C_{5} \omega_{2}^{5}-C_{1} \omega_{2} \\
& \left.+1 \omega_{2}\right) \cos \tau_{1} \omega_{2} \\
& \left.+D_{4} \omega_{2}^{4}-D_{0}\right) \sin \tau_{1} \omega_{2} \\
&
\end{aligned}
$$

Thus,

$\cos \tau_{2} \omega_{2}$

$$
=\frac{L_{52}\left(\omega_{2}\right) \times L_{56}\left(\omega_{2}\right)+L_{53}\left(\omega_{2}\right) \times L_{54}\left(\omega_{2}\right)}{L_{51}\left(\omega_{2}\right) \times L_{54}\left(\omega_{2}\right)+L_{52}\left(\omega_{2}\right) \times L_{55}\left(\omega_{2}\right)},
$$

$\sin \tau_{2} \omega_{2}$

$$
=\frac{L_{51}\left(\omega_{2}\right) \times L_{56}\left(\omega_{2}\right)-L_{53}\left(\omega_{2}\right) \times L_{55}\left(\omega_{2}\right)}{L_{51}\left(\omega_{2}\right) \times L_{54}\left(\omega_{2}\right)+L_{52}\left(\omega_{2}\right) \times L_{55}\left(\omega_{2}\right)} .
$$

Then, we get

$$
\cos ^{2} \tau_{2} \omega_{2}+\sin ^{2} \tau_{2} \omega_{2}=1
$$

If $\left(H_{51}\right)$ holds, then there exists $\omega_{20}^{*}>0$ such that (35) has a pair of purely imaginary roots $\pm i \omega_{20}^{*}$. For $\omega_{20}^{*}$,

$$
\begin{aligned}
\tau_{20}^{*} & =\frac{1}{\omega_{20}^{*}} \\
& \times \arccos \left\{\frac{L_{52}\left(\omega_{20}^{*}\right) \times L_{56}\left(\omega_{20}^{*}\right)+L_{53}\left(\omega_{20}^{*}\right) \times L_{54}\left(\omega_{20}^{*}\right)}{L_{51}\left(\omega_{20}^{*}\right) \times L_{54}\left(\omega_{20}^{*}\right)+L_{52}\left(\omega_{20}^{*}\right) \times L_{55}\left(\omega_{20}^{*}\right)}\right\} .
\end{aligned}
$$

Differentiating (25) with respect to $\tau_{2}$, one can get

$$
\left[\frac{d \lambda}{d \tau_{2}}\right]^{-1}=\frac{F_{51}(\lambda)}{F_{52}(\lambda)}-\frac{\tau_{2}}{\lambda}
$$

where

$$
\begin{aligned}
F_{51} & (\lambda)=5 C_{5} \lambda^{4}+4 C_{4} \lambda^{3}+3 C_{3} \lambda^{2}+2 C_{2} \lambda+C_{1} \\
& +\left(\left(4 D_{4}-\tau_{1} D_{3}\right) \lambda^{3}-\tau_{1} D_{4} \lambda^{4}+\left(3 D_{3}-\tau_{1} D_{2}\right) \lambda^{2}\right. \\
& \left.+\left(2 D_{2}-\tau_{1} D_{1}\right) \lambda+D_{1}-\tau_{1} D_{0}\right) e^{-\lambda \tau_{1}} \\
& +\left(\left(3 H_{3}-2 \tau_{1} H_{2}\right) \lambda^{2}-2 \tau_{1} H_{3} \lambda^{3}\right. \\
& \left.+2\left(H_{2}-\tau_{1} H_{1}\right) \lambda+H_{1}-2 \tau_{1} H_{0}\right) e^{-2 \lambda \tau_{1}}+\left(4 G_{4} \lambda^{3}\right. \\
& \left.+3 G_{3} \lambda^{2}+2 G_{2} \lambda+G_{1}\right) e^{-\lambda \tau_{2}}+\left(6 \lambda^{5}+5 A_{5} \lambda^{4}\right. \\
& \left.+4 A_{4} \lambda^{3}+3 A_{3} \lambda^{2}+2 A_{2} \lambda+A_{1}\right) e^{\lambda \tau_{2}} \\
& +\left(\left(5 B_{5}-\tau_{1} B_{4}\right) \lambda^{4}-\tau_{1} B_{5} \lambda^{5}+\left(4 B_{4}-\tau_{1} B_{3}\right) \lambda^{3}\right. \\
& \left.+\left(3 B_{3}-\tau_{1} B_{2}\right) \lambda^{2}+\left(2 B_{2}-\tau_{1} B_{1}\right) \lambda+B_{1}-\tau_{1} B_{0}\right) \\
& +e^{\lambda\left(\tau_{2}-\tau_{1}\right)}+\left(\left(3 E_{3}-\tau_{1} E_{2}\right) \lambda^{2}-\tau_{1} E_{3} \lambda^{3}\right. \\
& \left.+\left(2 E_{2}-\tau_{1} E_{1}\right) \lambda+E_{1}-\tau_{1} E_{0}\right) e^{-\lambda\left(\tau_{2}+\tau_{1}\right)} \\
& +\left(\left(4 F_{4}-2 \tau_{1} F_{3}\right) \lambda^{3}-2 \tau_{1} F_{4} \lambda^{4}+\left(3 F_{3}-2 \tau_{1} F_{2}\right) \lambda^{2}\right. \\
& \left.+\left(2 F_{2}-2 \tau_{1} F_{1}\right) \lambda+F_{1}-2 \tau_{1} F_{0}\right) e^{\lambda\left(\tau_{2}-2 \tau_{1}\right)} \\
& +\left(2\left(I_{2}-\tau_{1} I_{1}\right) \lambda-2 \tau_{1} I_{2} \lambda^{2}+I_{1}-2 \tau_{1} I_{0}\right) e^{\lambda\left(\tau_{2}+2 \tau_{1}\right)}, \\
& \left.+F_{1} \lambda^{2}+F_{0} \lambda\right) e^{\lambda\left(\tau_{2}-2 \tau_{1}\right)}-\left(B_{5} \lambda^{6}+B_{3} \lambda_{3} \lambda^{5}+B_{3} \lambda^{4} \lambda^{2}+B_{0} \lambda\right) e^{\lambda\left(\tau_{2}-\tau_{1}\right)}-\left(\lambda^{7}+A_{5} \lambda^{6}\right. \\
F_{52} & +(\lambda)=\left(G_{4} \lambda^{5}+\lambda_{3} \lambda^{4}+A_{0} \lambda\right) e^{\lambda \tau_{2}} . \\
+ & \left(E_{3} \lambda^{4}+E_{2} \lambda^{3}+E_{1} \lambda^{2}+E_{0} \lambda\right) e^{-\lambda\left(\tau_{2}+\tau_{1}\right)}+\left(I_{2} \lambda^{3}\right. \\
+ & \left.I_{1} \lambda\right) e^{-\lambda \tau_{2}}
\end{aligned}
$$


Thus,

$$
\operatorname{Re}\left[\frac{d \lambda}{d \tau_{2}}\right]_{\lambda=i \omega_{20}^{*}}^{-1}=\frac{G_{3 R} \times H_{3 R}+G_{3 I} \times H_{3 I}}{H_{3 R}^{2}+H_{3 I}^{2}} .
$$

Therefore, we know that if condition $\left(H_{52}\right) G_{5 R} \times H_{5 R}+$ $G_{5 I} \times H_{5 I} \neq 0$ holds, then $\operatorname{Re}\left[d \lambda / d \tau_{2}\right]_{\lambda=i \omega_{20}^{*}} \neq 0$. Then, we have the following results.

Theorem 4. Let $\tau_{1} \in\left(0, \tau_{10}\right)$ and suppose that conditions $\left(H_{1}\right)$, $\left(H_{51}\right)$, and $\left(H_{52}\right)$ hold for system (2). The viral equilibrium $P_{*}\left(S_{*}, E_{*}, I_{*}, Q_{*}, R_{*}, V_{*}\right)$ is locally asymptotically stable when $\tau_{2} \in\left[0, \tau_{20}^{*}\right)$ and a Hopf bifurcation occurs at the viral equilibrium $P_{*}\left(S_{*}, E_{*}, I_{*}, Q_{*}, R_{*}, V_{*}\right)$ when $\tau_{2}=\tau_{20}^{*}$.

\section{Properties of the Hopf Bifurcation}

In this section, we shall investigate direction and stability of the Hopf bifurcation under the case where $\tau_{1} \in\left(0, \tau_{10}\right)$ and $\tau_{2}>0$. Set $u_{1}(t)=S(t)-S_{*}, u_{2}(t)=E(t)-E_{*}, u_{3}(t)=I(t)-I_{*}$, $u_{4}(t)=Q(t)-Q_{*}, u_{5}(t)=R(t)-R_{*}, u_{6}(t)=V(t)-V_{*}$, and $t \rightarrow$ $\left(t / \tau_{2}\right)$. For convenience, we assume that $\tau_{1}^{*} \in\left(0, \tau_{10}\right)<\tau_{20}^{*}$ throughout this section. Then, system (2) becomes functional differential equations in $C=C\left([-1,0], R^{6}\right)$ :

$$
\dot{u}(t)=L_{\mu} u_{t}+F\left(\mu, u_{t}\right) \text {, }
$$

with

$$
\begin{gathered}
L_{\mu} \phi=\left(\tau_{20}^{*}+\mu\right)\left(A \phi(0)+B \phi\left(-\frac{\tau_{1}^{*}}{\tau_{20}^{*}}\right)+C \phi(-1)\right) \\
F(\mu, \phi)=\left(\tau_{20}^{*}+\mu\right)\left(\begin{array}{c}
-\beta \phi_{1}(0) \phi_{3}(0) \\
\beta \phi_{1}(0) \phi_{3}(0) \\
0 \\
0 \\
0 \\
0
\end{array}\right),
\end{gathered}
$$

where

$$
\begin{aligned}
A & =\left(\begin{array}{cccccc}
a_{1} & 0 & a_{2} & 0 & 0 & 0 \\
a_{3} & a_{4} & a_{5} & 0 & 0 & 0 \\
0 & a_{6} & a_{7} & 0 & 0 & 0 \\
0 & 0 & a_{8} & a_{9} & 0 & 0 \\
0 & 0 & 0 & 0 & a_{10} & 0 \\
a_{11} & 0 & 0 & 0 & 0 & a_{12}
\end{array}\right), \\
B & =\left(\begin{array}{cccccc}
0 & 0 & 0 & 0 & 0 & 0 \\
0 & 0 & 0 & 0 & 0 & 0 \\
0 & 0 & b_{1} & 0 & 0 & 0 \\
0 & 0 & 0 & b_{2} & 0 & 0 \\
0 & 0 & b_{3} & b_{4} & 0 & 0 \\
0 & 0 & 0 & 0 & 0 & 0
\end{array}\right)
\end{aligned}
$$

$$
C=\left(\begin{array}{cccccc}
0 & 0 & 0 & 0 & c_{1} & c_{2} \\
0 & 0 & 0 & 0 & 0 & 0 \\
0 & 0 & 0 & 0 & 0 & 0 \\
0 & 0 & 0 & 0 & 0 & 0 \\
0 & 0 & 0 & c_{3} & 0 & 0 \\
0 & 0 & 0 & 0 & 0 & c_{4}
\end{array}\right)
$$

Based on the Riesz representation theorem, there exists a $6 \times 6$ function $\eta(\theta, \mu):[-1,0] \rightarrow R^{6 \times 6}$ such that

$$
L_{\mu} \phi=\int_{-1}^{0} d \eta(\theta, \mu) \phi(\theta), \quad \phi \in C .
$$

In fact, we choose

$$
\begin{aligned}
\eta(\theta, \mu) & \begin{cases}\left(\tau_{20}^{*}+\mu\right)(A+B+B), & \theta=0, \\
\left(\tau_{20}^{*}+\mu\right)(B+C), & \theta \in\left[-\frac{\tau_{1}^{*}}{\left.\tau_{20}^{*}, 0\right),}\right. \\
\left(\tau_{20}^{*}+\mu\right) B, & \theta \in\left(-1,-\frac{\tau_{1}^{*}}{\tau_{20}^{*}}\right), \\
0, & \theta=-1 .\end{cases}
\end{aligned}
$$

For $\phi \in C\left([-1,0], R^{6}\right)$, we define

$$
\begin{aligned}
& A(\mu) \phi= \begin{cases}\frac{d \phi(\theta)}{d \theta}, & -1 \leq \theta<0, \\
\int_{-1}^{0} d \eta(\theta, \mu) \phi(\theta), & \theta=0,\end{cases} \\
& R(\mu) \phi= \begin{cases}0, & -1 \leq \theta<0, \\
F(\mu, \phi), & \theta=0 .\end{cases}
\end{aligned}
$$

Then, system (53) becomes

$$
\dot{u}(t)=A(\mu) u_{t}+R(\mu) u_{t},
$$

where $u_{t}(\theta)=u(t+\theta)$ for $\theta \in[-1,0]$.

Define $A^{*}$ as follows:

$$
A^{*}(\varphi)= \begin{cases}-\frac{d \varphi(s)}{d s}, & 0<s \leq 1, \\ \int_{-1}^{0} d \eta^{T}(s, 0) \varphi(-s), & s=0,\end{cases}
$$

and a bilinear form

$$
\begin{aligned}
\langle\varphi(s), \phi(\theta)\rangle= & \bar{\varphi}(0) \phi(0) \\
& -\int_{\theta=-1}^{0} \int_{\xi=0}^{\theta} \bar{\varphi}(\xi-\theta) d \eta(\theta) \phi(\xi) d \xi,
\end{aligned}
$$


Let $q(\theta)=\left(1, q_{2}, q_{3}, q_{4}, q_{5}, q_{6}\right)^{T} e^{i \omega_{20}^{*} \tau_{20}^{*} \theta}$ be the eigenvector of $A(0)$ with $+i \omega_{20}^{*} \tau_{20}^{*}$ and let $q^{*}(s)=D\left(1, q_{2}^{*}, q_{3}^{*}\right.$, $\left.q_{4}^{*}, q_{5}^{*}\right) e^{i \omega_{20}^{*} \tau_{20}^{*} s}$ be the eigenvector of $A^{*}(0)$ with $-i \omega_{20}^{*} \tau_{20}^{*}$. Then, according to the definition of $A(0)$ and $A^{*}(0)$, we obtain

$$
\begin{aligned}
& q_{2}=\frac{a_{3}+a_{5} q_{3}}{i \omega_{20}^{*}-a_{3}}, \\
& q_{3}=\frac{a_{3} a_{6}}{\left(i \omega_{20}^{*}-a_{3}\right)\left(i \omega_{20}^{*}-a_{7}-b_{1} e^{-i \tau_{1}^{*} \omega_{20}^{*}}\right)-a_{5} a_{6}}, \\
& q_{4}=\frac{a_{8} q_{3}}{i \omega_{20}^{-} a_{9}-b_{2} e^{-i \tau_{1}^{*} \omega_{20}^{*}}}, \\
& q_{5}=\frac{i \omega_{20}^{*}-a_{1}-a_{2} q_{3}-c_{2} e^{-i \tau_{20}^{*} \omega_{20}^{*}}}{c_{1} e^{-i \tau_{20}^{*} \omega_{20}^{*}}}, \\
& q_{6}=\frac{i \omega_{20}^{*}-a_{11}}{a_{12}+c_{4} e^{-i \tau_{20}^{*} \omega_{20}^{*}}}, \\
& q_{2}^{*}=-\frac{i \omega_{20}^{*}+a_{1}}{a_{3}}, \\
& q_{3}^{*}=\frac{\left(i \omega_{20}^{*}+a_{1}\right)\left(i \omega_{20}^{*}+a_{4}\right)}{a_{3} a_{6}}, \\
& q_{4}^{*}=-\frac{\left(i \omega_{20}^{*}+a_{7}+b_{1} e^{i \tau_{1}^{*} \omega_{20}^{*}}\right) q_{3}}{a_{8}} \\
& +\frac{a_{2} a_{3}+\left(i \omega_{20}^{*}+a_{1}\right) a_{5}}{a_{3} a_{8}} \\
& +\frac{b_{3}\left(i \omega_{20}^{*}+a_{9}+b_{2} e^{i \tau_{1}^{*} \omega_{20}^{*}}\right)}{a_{8} b_{4}}, \\
& q_{5}=-\frac{\left(i \omega_{20}^{*}+a_{9}+b_{2} e^{i \tau_{1}^{*} \omega_{20}^{*}}\right) q_{4}}{b_{4} e^{i \tau_{1}^{*} \omega_{20}^{*}}}, \\
& q_{6}^{*}=-\frac{c_{2} e^{i \tau_{20}^{*} \omega_{20}^{*}}}{i \omega_{20}^{*}+a_{12}+c_{4} e^{i \tau_{20}^{*} \omega_{20}^{*}}} .
\end{aligned}
$$

In addition, from (61), we have

$$
\begin{aligned}
& \left\langle q^{*}(s), q(\theta)\right\rangle=\bar{q}(0) q(0) \\
& \quad-\int_{-1}^{0} \int_{\xi=0}^{\theta} \bar{q}^{*}(\xi-\theta) d \eta(\theta) q(\xi) d \xi \\
& \quad=\bar{D}\left(1, \bar{q}_{2}^{*}, \bar{q}_{3}^{*}, \bar{q}_{4}^{*}, \bar{q}_{5}^{*}, \bar{q}_{6}^{*}\right)\left(1, q_{2}, q_{3}, q_{4}, q_{5}, q_{6}\right)^{T} \\
& \quad-\int_{-1}^{0} \int_{\xi=0}^{\theta} \bar{D}\left(1, \bar{q}_{2}^{*}, \bar{q}_{3}^{*}, \bar{q}_{4}^{*}, \bar{q}_{5}^{*}, \bar{q}_{6}^{*}\right)
\end{aligned}
$$

$$
\begin{aligned}
& \cdot e^{-i \tau_{1}^{*} \omega_{20}^{*}(\xi-\theta)} d \eta(\theta)\left(1, q_{2}, q_{3}, q_{4}, q_{5}, q_{6}\right)^{T} e^{i i \tau_{1}^{*} \omega_{20}^{*} \xi} d \xi \\
& -\int_{-1}^{0} \int_{\xi=0}^{\theta} \bar{D}\left(1, \bar{q}_{2}^{*}, \bar{q}_{3}^{*}, \bar{q}_{4}^{*}, \bar{q}_{5}^{*}, \bar{q}_{6}^{*}\right) \\
& \cdot e^{-i \tau_{20}^{*} \omega_{20}^{*}(\xi-\theta)} d \eta(\theta)\left(1, q_{2}, q_{3}, q_{4}, q_{5}, q_{6}\right)^{T} e^{i \tau_{20}^{*} \omega_{20}^{*} \xi} d \xi \\
& =\bar{D}\left[1+q_{2} \bar{q}_{2}^{*}+q_{3} \bar{q}_{3}^{*}+q_{4} \bar{q}_{4}^{*}+q_{5} \bar{q}_{5}^{*}+q_{6} \bar{q}_{6}^{*}\right. \\
& +\tau_{1}^{*} e^{i \tau_{1}^{*} \omega_{20}^{*}} \int_{-1}^{0}\left(1, \bar{q}_{2}^{*}, \bar{q}_{3}^{*}, \bar{q}_{4}^{*}, \bar{q}_{5}^{*}, \bar{q}_{6}^{*}\right) \\
& \cdot B\left(1, q_{2}, q_{3}, q_{4}, q_{5}, q_{6}\right)^{T} \\
& +\tau_{1}^{*} e^{i \tau_{2}^{*} \omega_{20}^{*}} \int_{-1}^{0}\left(1, \bar{q}_{2}^{*}, \bar{q}_{3}^{*}, \bar{q}_{4}^{*}, \bar{q}_{5}^{*}, \bar{q}_{6}^{*}\right) \\
& \left.\cdot C\left(1, q_{2}, q_{3}, q_{4}, q_{5}, q_{6}\right)^{T}\right]=\bar{D}\left[1+q_{2} \bar{q}_{2}^{*}+q_{3} \bar{q}_{3}^{*}\right. \\
& +q_{4} \bar{q}_{4}^{*}+q_{5} \bar{q}_{5}^{*}+q_{6} \bar{q}_{6}^{*} \\
& +\tau_{1}^{*} e^{-i \tau_{1}^{*} \omega_{20}^{*}}\left(b_{3}\left(b_{1} \bar{q}_{3}^{*}+b_{3} \bar{q}_{5}^{*}\right)+b_{4}\left(b_{2} \bar{q}_{4}^{*}+b_{4} \bar{q}_{5}^{*}\right)\right) \\
& \left.+\tau_{20}^{*} e^{-i \tau_{20}^{*} \omega_{20}^{*}}\left(c_{1} \bar{q}_{5}^{*}+c_{2} \bar{q}_{6}^{*}+c_{3} q_{5} \bar{q}_{5}^{*}+c_{4} q_{6} \bar{q}_{6}^{*}\right)\right] .
\end{aligned}
$$

Thus, we can choose

$$
\begin{aligned}
\bar{D} & =\left[1+q_{2} \bar{q}_{2}^{*}+q_{3} \bar{q}_{3}^{*}+q_{4} \bar{q}_{4}^{*}+q_{5} \bar{q}_{5}^{*}+q_{6} \bar{q}_{6}^{*}\right. \\
& +\tau_{1}^{*} e^{-i \tau_{1}^{*} \omega_{20}^{*}}\left(b_{3}\left(b_{1} \bar{q}_{3}^{*}+b_{3} \bar{q}_{5}^{*}\right)+b_{4}\left(b_{2} \bar{q}_{4}^{*}+b_{4} \bar{q}_{5}^{*}\right)\right) \\
& \left.+\tau_{20}^{*} e^{-i \tau_{20}^{*} \omega_{20}^{*}}\left(c_{1} \bar{q}_{5}^{*}+c_{2} \bar{q}_{6}^{*}+c_{3} q_{5} \bar{q}_{5}^{*}+c_{4} q_{6} \bar{q}_{6}^{*}\right)\right]^{-1},
\end{aligned}
$$

such that $\left\langle q^{*}, q\right\rangle=1,\left\langle q^{*}, \bar{q}\right\rangle=0$.

Then, using the algorithms from Hassard et al. [25] and the similar computation process in [26-29], we obtain

$$
\begin{aligned}
g_{20} & =2 \beta \tau_{20}^{*} \bar{D} q_{3}\left(\bar{q}_{2}^{*}-1\right), \\
g_{11} & =\beta \tau_{20}^{*} \bar{D} \operatorname{Re}\left\{q_{3}\right\}\left(\bar{q}_{2}^{*}-1\right), \\
g_{02} & =2 \beta \tau_{20}^{*} \bar{D} \bar{q}_{3}\left(\bar{q}_{2}^{*}-1\right), \\
g_{21} & =2 \beta \tau_{20}^{*} \bar{D}\left(\bar{q}_{2}^{*}-1\right)\left(W_{11}^{(1)}(0) q_{3}+\frac{1}{2} W_{20}^{(1)}(0) \bar{q}_{3}\right. \\
& \left.+W_{11}^{(3)}(0)+\frac{1}{2} W_{20}^{(3)}(0)\right),
\end{aligned}
$$

with

$$
\begin{aligned}
& W_{20}(\theta)=\frac{i g_{20} q(0)}{\tau_{20}^{*} \omega_{20}^{*}} e^{i \tau_{20}^{*} \omega_{20}^{*} \theta}+\frac{i \bar{g}_{02} \bar{q}(0)}{3 \tau_{20}^{*} \omega_{20}^{*}} e^{-i \tau \tau_{20}^{*} \omega_{20}^{*} \theta}+E_{1} e^{2 i \tau_{20}^{*} \omega_{20}^{*} \theta}, \\
& W_{11}(\theta)=-\frac{i g_{11} q(0)}{\tau_{20}^{*} \omega_{20}^{*}} e^{i \tau_{20}^{*} \omega_{20}^{*} \theta}+\frac{i \bar{g}_{11} \bar{q}(0)}{\tau_{20}^{*} \omega_{20}^{*}} e^{-i \tau_{20}^{*} \omega_{20}^{*} \theta}+E_{2},
\end{aligned}
$$




$$
\begin{aligned}
& E_{1}=2\left(\begin{array}{cccccc}
a_{1}^{\prime} & 0 & -a_{2} & 0 & -c_{1} e^{-2 i \tau_{20}^{*} \omega_{20}^{*}} & -c_{2} e^{-2 i \tau_{20}^{*} \omega_{20}^{*}} \\
-a_{3} & a_{2}^{\prime} & -a_{5} & 0 & 0 & 0 \\
0 & -a_{6} & a_{3}^{\prime} & 0 & 0 & 0 \\
0 & 0 & -a_{8} & a_{4}^{\prime} & 0 & 0 \\
0 & 0 & -b_{3} e^{-2 i \tau_{1}^{*} \omega_{20}^{*}} & -b_{4} e^{-2 i \tau_{1}^{*} \omega_{20}^{*}} & a_{5}^{\prime} & 0 \\
-a_{11} & 0 & 0 & 0 & 0 & a_{6}^{\prime}
\end{array}\right)^{-1} \times\left(\begin{array}{c}
-\beta q_{3} \\
\beta q_{3} \\
0 \\
0 \\
0 \\
0
\end{array}\right), \\
& E_{2}=-\left(\begin{array}{cccccc}
a_{1} & 0 & a_{2} & 0 & c_{1} & c_{2} \\
a_{3} & a_{4} & a_{5} & 0 & 0 & 0 \\
0 & a_{6} & a_{7}+b_{1} & 0 & 0 & 0 \\
0 & 0 & a_{8} & a_{9}+b_{2} & 0 & 0 \\
0 & 0 & b_{3} & b_{4} & a_{10}+c_{3} & 0 \\
a_{11} & 0 & 0 & 0 & 0 & a_{12}+c_{4}
\end{array}\right)^{-1} \times\left(\begin{array}{c}
-\beta \operatorname{Re}\left\{q_{3}\right\} \\
\beta \operatorname{Re}\left\{q_{3}\right\} \\
0 \\
0 \\
0 \\
0
\end{array}\right) \times
\end{aligned}
$$

where

$$
\begin{aligned}
& a_{1}^{\prime}=2 i \omega_{20}^{*}-a_{1}, \\
& a_{2}^{\prime}=2 i \omega_{20}^{*}-a_{4}, \\
& a_{3}^{\prime}=2 i \omega_{20}^{*}-a_{7}-b_{1} e^{-2 i \tau_{1}^{*} \omega_{20}^{*},} \\
& a_{4}^{\prime}=2 i \omega_{20}^{*}-a_{9}-b_{2} e^{-2 i \tau_{1}^{*} \omega_{20}^{*},} \\
& a_{5}^{\prime}=2 i \omega_{20}^{*}-a_{10}-c_{3} e^{-2 i \tau_{20}^{*} \omega_{20}^{*},} \\
& a_{6}^{\prime}=2 i \omega_{20}^{*}-a_{12}-c_{4} e^{-2 i \tau_{20}^{*} \omega_{20}^{*}} .
\end{aligned}
$$

Then, we can get the following coefficients:

$$
\begin{aligned}
C_{1}(0)= & \frac{i}{2 \tau_{20}^{*} \omega_{20}^{*}}\left(g_{11} g_{20}-2\left|g_{11}\right|^{2}-\frac{\left|g_{02}\right|^{2}}{3}\right) \\
& +\frac{g_{21}}{2}, \\
\mu_{2}= & -\frac{\operatorname{Re}\left\{C_{1}(0)\right\}}{\operatorname{Re}\left\{\lambda^{\prime}\left(\tau_{20}^{*}\right)\right\}}, \\
\rho_{2}= & 2 \operatorname{Re}\left\{C_{1}(0)\right\}, \\
T_{2}= & -\frac{\operatorname{Im}\left\{C_{1}(0)\right\}+\mu_{2} \operatorname{Im}\left\{\lambda^{\prime}\left(\tau_{20}^{*}\right)\right\}}{\tau_{20}^{*} \omega_{20}^{*}} .
\end{aligned}
$$

Thus, we have the following results.

Theorem 5. The sign of $\mu_{2}$ determines direction of the Hopf bifurcation: if $\mu_{2}>0\left(\mu_{2}<0\right)$, then the Hopf bifurcation is supercritical (subcritical); the sign of $\rho_{2}$ determines stability of the bifurcating periodic solutions: if $\rho_{2}<0\left(\rho_{2}>0\right)$, then the bifurcating periodic solutions are stable (unstable); the sign of $T_{2}$ determines period of the bifurcating solutions: if $T_{2}>0$
$\left(T_{2}<0\right)$, then the period of the bifurcating periodic solutions increases (decreases).

\section{Numerical Simulation}

In this section, we present some numerical results of system (2) in order to validate the analytical predictions obtained in Sections 2 and 3 . We choose a set of parameters as follows: $A=100, \beta=0.009, d=0.05, \rho=0.65, \theta=0.05, \chi=0.55$, $\gamma=0.45, \alpha=0.035, \delta=0.1, \eta=0.35$, and $\varepsilon=0.07$, and consider the following special case of (2):

$$
\begin{aligned}
\frac{d S(t)}{d t}= & 100-0.009 S(t) I(t)-0.05 S(t)-0.65 S(t) \\
& +0.05 R\left(t-\tau_{2}\right)+0.55 V\left(t-\tau_{2}\right), \\
\frac{d E(t)}{d t}= & 0.009 S(t) I(t)-0.05 E(t)-0.45 E(t), \\
\frac{d I(t)}{d t}= & 0.45 E(t)-0.05 I(t)-0.035 I(t)-0.1 I(t) \\
& -0.35 I\left(t-\tau_{1}\right), \\
\frac{d Q(t)}{d t}= & \delta I(t)-0.05 Q(t)-0.035 Q(t) \\
& -0.07 Q\left(t-\tau_{1}\right), \\
\frac{d R(t)}{d t}= & 0.07 Q\left(t-\tau_{1}\right)-0.05 R(t)-0.05 R\left(t-\tau_{2}\right) \\
& +0.35 I\left(t-\tau_{1}\right), \\
\frac{d V(t)}{d t}= & 0.65 S(t)-0.05 \mathrm{~V}(t)-0.55 \mathrm{~V}\left(t-\tau_{2}\right),
\end{aligned}
$$

from which we can get the unique viral equilibrium $P_{*}(66.0494,277.7978,233.6617,150.7495,923.3406,71.7439)$. 


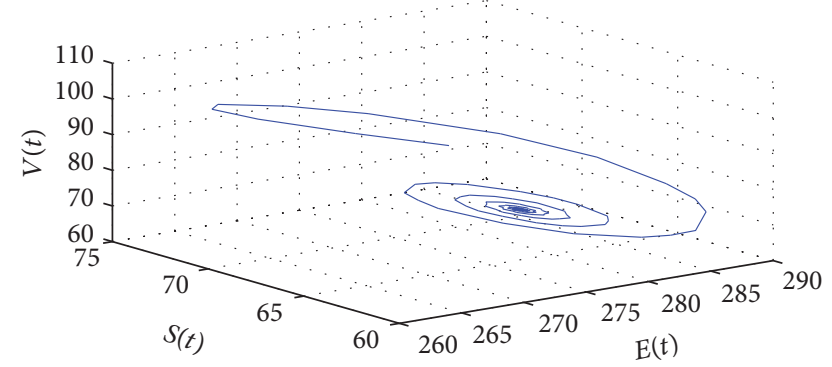

FIgURE 1: The projection of the phase portrait of system (69) in $(S, E, V)$-space with $\tau_{1}=3.605$.

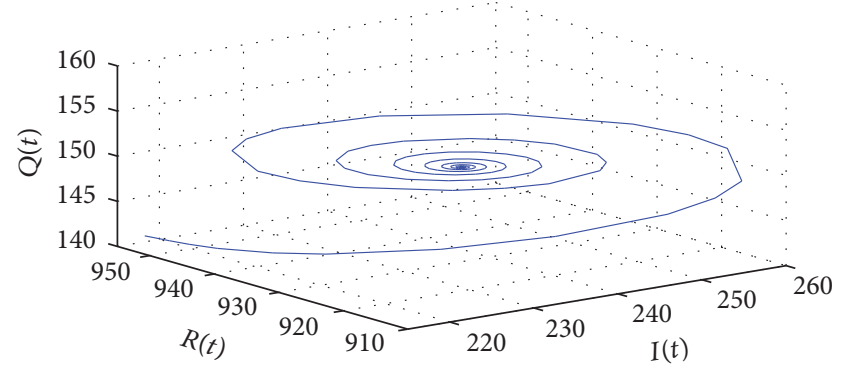

FIGURE 2: The projection of the phase portrait of system (69) in $(I, Q, R)$-space with $\tau_{1}=3.605$.

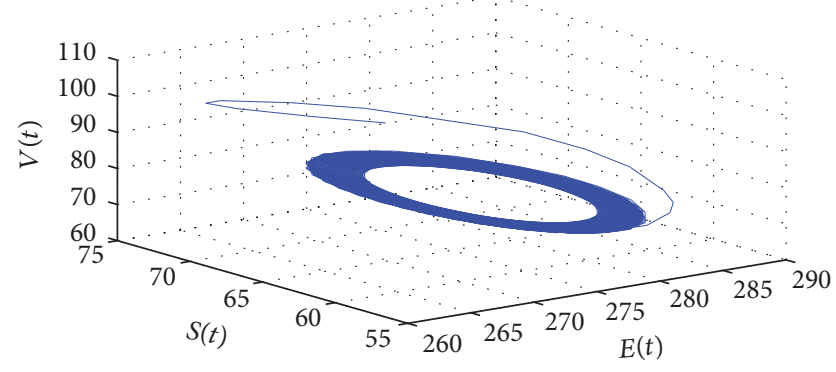

FIGURE 3: The projection of the phase portrait of system (69) in $(S, E, V)$-space with $\tau_{1}=4.60$.

It can be easily verified that condition $\left(H_{1}\right)$ is satisfied when $\tau_{1}=\tau_{2}=0$.

By computation, we have $\omega_{10}=0.8554$ and $\tau_{10}=$ 4.1056. Then, we get $\lambda^{\prime}\left(\tau_{10}\right)=2.3686+i 1.0212$. Thus, we know that conditions $\left(H_{21}\right)$ and $\left(H_{22}\right)$ hold. We can conclude that all roots that cross the imaginary axis at $i \omega_{10}$ cross from left to right as $\tau_{1}$ increases by the theory in [22]. According to Theorem 1, $P_{*}(66.0494$, $277.7978,233.6617,150.7495,923.3406,71.7439)$ is asymptotically stable when $\tau_{1} \in\left(0, \tau_{10}\right)$. This property can be illustrated by Figures 1 and 2 . In this case, spreading law of the computer viruses can be predicted and the viruses can be controlled and eliminated. However, once the value of $\tau_{1}$ passes through the critical value $\tau_{10}, P_{*}(66.0494$, $277.7978,233.6617,150.7495,923.3406,71.7439)$ loses its stability and a Hopf bifurcation occurs, which can be shown in Figures 3 and 4. The occurrence of a Hopf bifurcation means that the state of computer viruses propagation changes

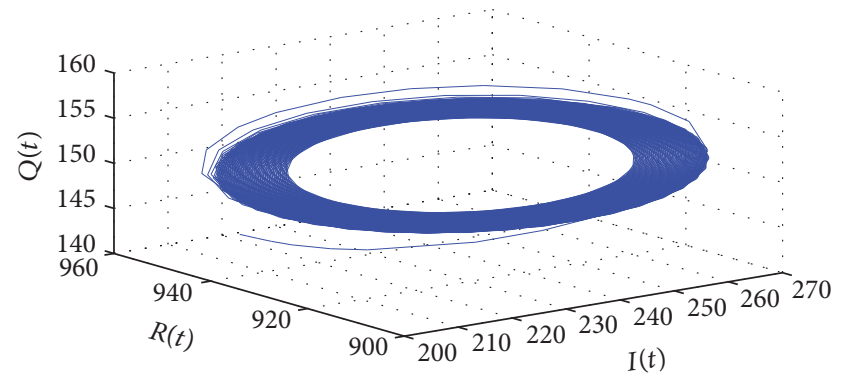

FIGURE 4: The projection of the phase portrait of system (69) in $(I, Q, R)$-space with $\tau_{1}=4.60$.

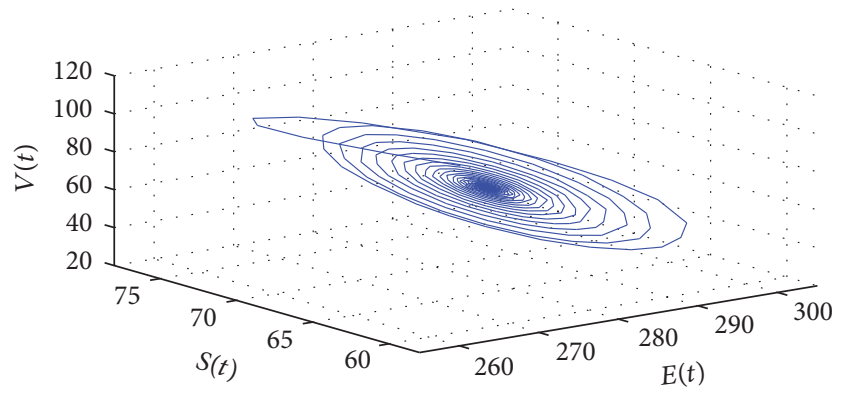

FIGURE 5: The projection of the phase portrait of system (69) in $(S, E, V)$-space with $\tau_{2}=3.65$.

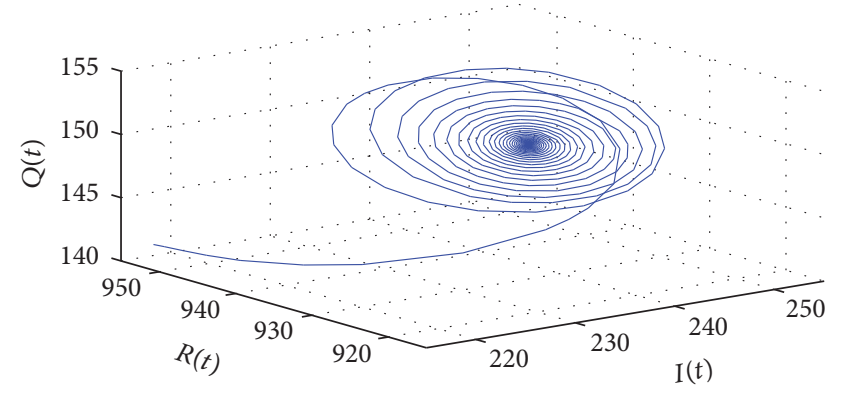

FIGURE 6: The projection of the phase portrait of system (69) in $(I, Q, R)$-space with $\tau_{2}=3.65$.

from the viral equilibrium point to a limit cycle. This makes spreading of the computer viruses be out of control.

Similarly, we have the following: $\omega_{20}=1.8255$ and $\tau_{20}=$ 3.7424 when $\tau_{1}=0$ and $\tau_{2}>0 ; \omega_{10}^{*}=0.9665$ and $\tau_{10}^{*}=3.1862$ when $\tau_{1}>0$ and $\tau_{2}=2.25 \in\left(0, \tau_{20}\right) ; \omega_{20}^{*}=2.4217$ and $\tau_{20}^{*}=3.0254$ when $\tau_{2}>0$ and $\tau_{1}=2.45 \in\left(0, \tau_{10}\right)$. The corresponding phase plots are shown in Figures 5-8, Figures 9-12, and Figures 13-16, respectively. In addition, for $\tau_{2}>0$ and $\tau_{1}=2.45 \in\left(0, \tau_{10}\right)$, we have $C_{1}(0)=-17.2982+i 13.5056$ and $\lambda^{\prime}\left(\tau_{20}^{*}\right)=0.3796+i 2.0581$ by some complex computation. Based on (68), we get $\mu_{2}=45.5692>0, \rho_{2}=-34.5964<0$, and $T_{2}=-14.6441<0$. Therefore, the Hopf bifurcation is supercritical, the bifurcating periodic solutions are stable, and the period of the bifurcating periodic solutions decreases.

According to the numerical simulation results, we know that the time delay should remain less than the corresponding threshold in order to control and predict the viruses' propagation by decreasing the period that antivirus software uses 


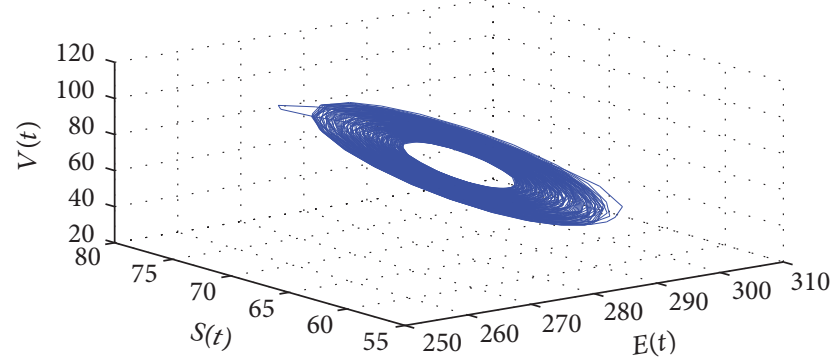

FIgURE 7: The projection of the phase portrait of system (69) in $(S, E, V)$-space with $\tau_{2}=3.805$.

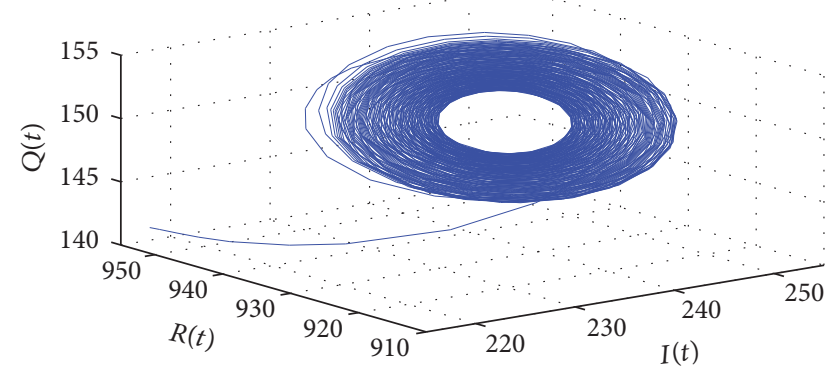

FIGURE 8: The projection of the phase portrait of system (69) in $(I, Q, R)$-space with $\tau_{2}=3.805$.

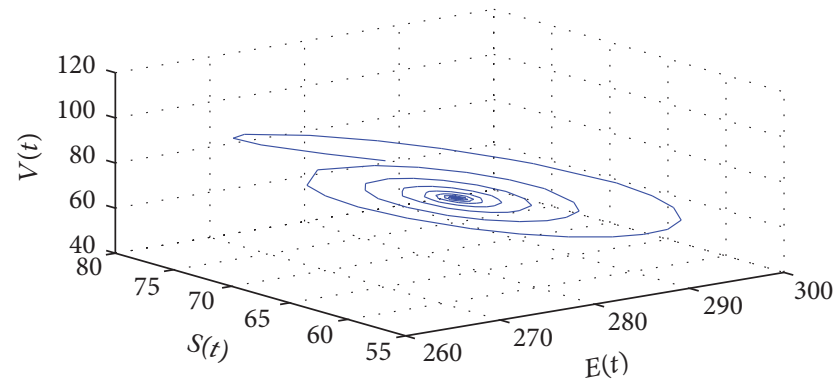

FIGURE 9: The projection of the phase portrait of system (69) in $(S, E, V)$-space with $\tau_{1}=2.86$ and $\tau_{2}=2.25 \in\left(0, \tau_{20}\right)$.

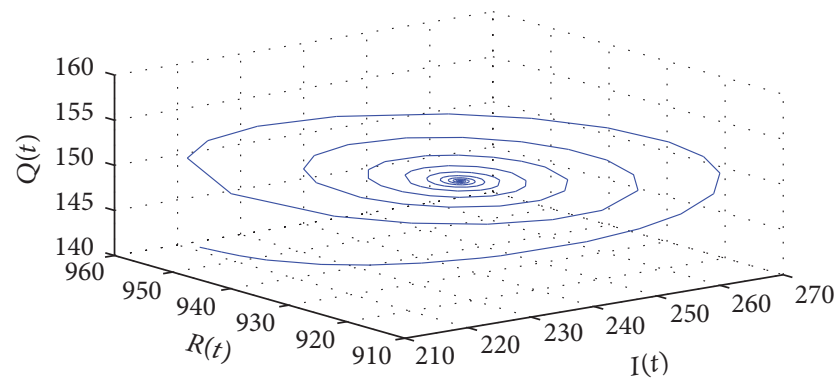

FIgURE 10: The projection of the phase portrait of system (69) in $(I, Q, R)$-space with $\tau_{1}=2.86$ and $\tau_{2}=2.25 \in\left(0, \tau_{20}\right)$.

to clean the computer viruses and the temporary immunity period of the recovered and the vaccinated computers. To this end, we can adjust the parameters of our proposed model in real-world networks, such as timely updating the antivirus software on computers, properly controlling the number of computers attached to the network, and timely

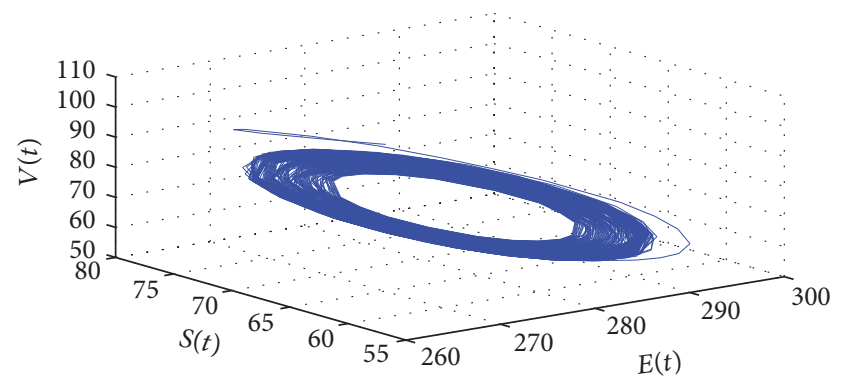

FIGURE 11: The projection of the phase portrait of system (69) in $(S, E, V)$-space with $\tau_{1}=3.574$ and $\tau_{2}=2.25 \in\left(0, \tau_{20}\right)$.

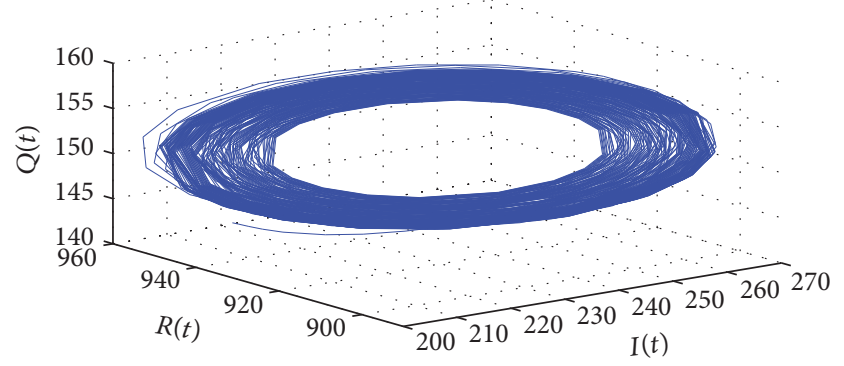

FIGURE 12: The projection of the phase portrait of system (69) in $(I, Q, R)$-space with $\tau_{1}=3.574$ and $\tau_{2}=2.25 \in\left(0, \tau_{20}\right)$.

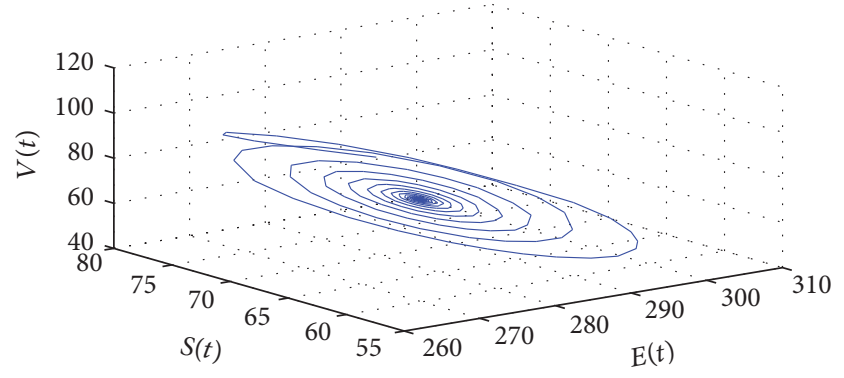

FIGURE 13: The projection of the phase portrait of system (69) in $(S, E, V)$-space with $\tau_{2}=2.862$ and $\tau_{1}=2.45 \in\left(0, \tau_{10}\right)$.

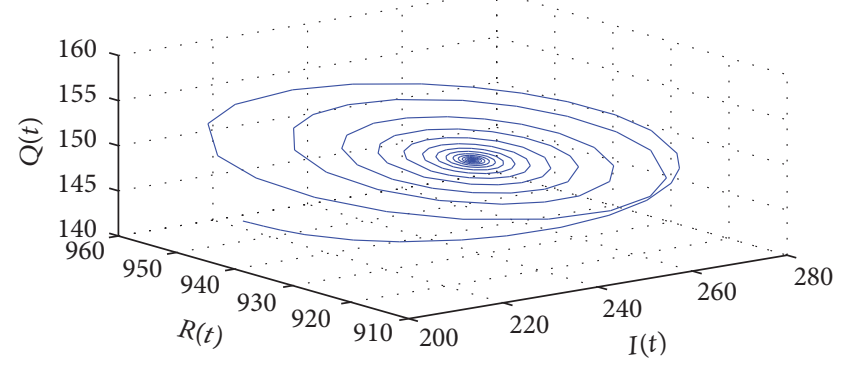

FIGURE 14: The projection of the phase portrait of system (69) in $(I, Q, R)$-space with $\tau_{2}=2.862$ and $\tau_{1}=2.45 \in\left(0, \tau_{10}\right)$.

disconnecting computers from the network when the connections are unnecessary. Of course, in the next step, we also need to collect large amount of relevant data and estimate the parameters involved in our proposed model through statistical analysis in real-world networks. Namely, we have to adjust the parameters in the model so as to control viruses' propagation effectively if it is necessary. 


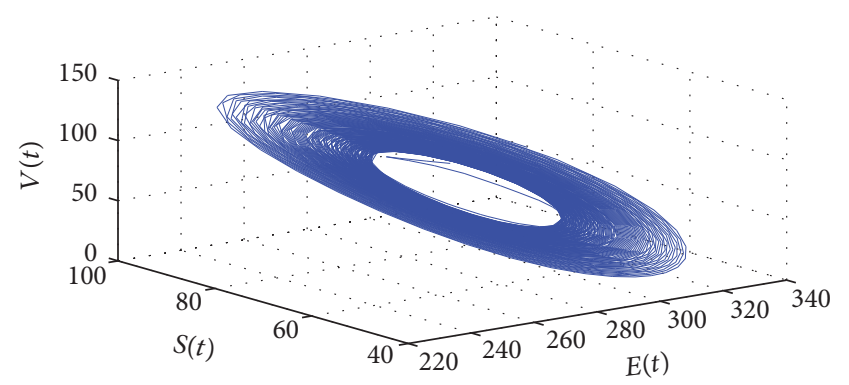

FIGURE 15: The projection of the phase portrait of system (69) in $(S, E, V)$-space with $\tau_{2}=3.225$ and $\tau_{1}=2.45 \in\left(0, \tau_{10}\right)$.

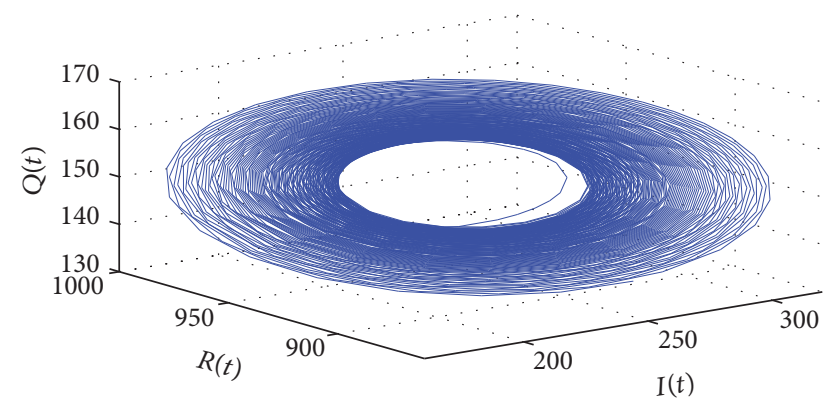

FIgURE 16: The projection of the phase portrait of system (69) in $(I, Q, R)$-space with $\tau_{2}=3.225$ and $\tau_{1}=2.45 \in\left(0, \tau_{10}\right)$.

\section{Conclusions}

It is definitely an interesting work to consider the effect of delays on dynamical systems, because a stability switch occurs even when an ignored delay is small for a dynamical system. Based on this fact, we introduce the time delay due to the period that antivirus software uses to clean the computer viruses in the infectious and quarantined computers $\left(\tau_{1}\right)$ and the time delay due to the temporary immunity period of the recovered and the vaccinated computers $\left(\tau_{2}\right)$ into the SEIQRS-V computer virus propagation model considered in [21]. We obtain some conditions for local stability and Hopf bifurcation occurring by analyzing distribution of roots of the associated characteristic equation.

By computation, there exists a corresponding critical value of the time delay below which system (2) is stable and above which system (2) is unstable. When the system is stable, the characteristics of the propagation of computer viruses can be easily predicted and then the computer viruses can get eliminated. Otherwise, the propagation of the computer viruses is out of control. Therefore, stability of the computer virus propagation system must be guaranteed in practice. In addition, we find that the effect of $\tau_{2}$ on system (2) is marked compared with $\tau_{1}$, because the critical value of $\tau_{2}$ is much smaller when we only consider it. At last, we have also derived the explicit formula which can determine direction and stability of the Hopf bifurcation under the case where $\tau_{1} \in\left(0, \tau_{10}\right)$ and $\tau_{2}>0$.

\section{Conflicts of Interest}

The authors declare that there are no conflicts of interest regarding the publication of this paper.

\section{Acknowledgments}

This work was supported by Natural Science Foundation of Anhui Province (nos. 1608085QF151, 1608085QF145, and 1708085MA17) and Natural Science Foundation of the Higher Education Institutions of Anhui Province (nos. KJ2014A006 and KJ2015A144).

\section{References}

[1] L.-X. Yang and X. Yang, "The effect of infected external computers on the spread of viruses: A Compartment Modeling Study," Physica A. Statistical Mechanics and its Applications, vol. 392, no. 24, pp. 6523-6535, 2013.

[2] P. Szor, The Art of Computer Virus Research and Defense, Addison-Wesley Education Publishers Inc., Boston, Mass, USA, 2005.

[3] H. Yuan and G. Chen, "Network virus-epidemic model with the point-to-group information propagation," Applied Mathematics and Computation, vol. 206, no. 1, pp. 357-367, 2008.

[4] B. K. Mishra and S. K. Pandey, "Dynamic model of worms with vertical transmission in computer network," Applied Mathematics and Computation, vol. 217, no. 21, pp. 8438-8446, 2011.

[5] L.-X. Yang, X. Yang, L. Wen, and J. Liu, "A novel computer virus propagation model and its dynamics," International Journal of Computer Mathematics, vol. 89, no. 17, pp. 2307-2314, 2012.

[6] J. Ren, X. Yang, Q. Zhu, L.-X. Yang, and C. Zhang, "A novel computer virus model and its dynamics," Nonlinear Analysis. Real World Applications, vol. 13, no. 1, pp. 376-384, 2012.

[7] C. Gan, X. Yang, W. Liu, Q. Zhu, and X. Zhang, "Propagation of computer virus under human intervention: a dynamical model," Discrete Dynamics in Nature and Society, vol. 2012, Article ID 106950, 8 pages, 2012.

[8] L. X. Yang, M. Draief, and X. F. Yang, "Heterogeneous virus propagation in networks: a theoretical study," Mathematical Methods in the Applied Sciences, vol. 40, no. 5, pp. 1396-1413, 2017.

[9] C. Zhang, Y. Zhao, Y. Wu, and S. Deng, "A stochastic dynamic model of computer viruses," Discrete Dynamics in Nature and Society, vol. 2012, Article ID 264874, 16 pages, 2012.

[10] A. Jafarabadi and M. A. Azgomi, "A stochastic epidemiological model for the propagation of active worms considering the dynamicity of network topology," Peer-to-Peer Networking and Applications, vol. 8, no. 6, pp. 1008-1022, 2015.

[11] J. Amador, "The stochastic SIRA model for computer viruses," Applied Mathematics and Computation, vol. 232, pp. 1112-1124, 2014.

[12] J. Amador and J. R. Artalejo, "Stochastic modeling of computer virus spreading with warning signals," Journal of the Franklin Institute. Engineering and Applied Mathematics, vol. 350, no. 5, pp. 1112-1138, 2013.

[13] Y. Yao, X.-W. Xie, H. Guo, G. Yu, F.-X. Gao, and X.-J. Tong, "Hopf bifurcation in an Internet worm propagation model with time delay in quarantine," Mathematical and Computer Modelling, vol. 57, no. 11-12, pp. 2635-2646, 2013. 
[14] J. Ren and Y. Xu, "Stability and bifurcation of a computer virus propagation model with delay and incomplete antivirus ability," Mathematical Problems in Engineering, vol. 2014, Article ID 475934, 2014

[15] J. Liu, "Hopf bifurcation in a delayed SEIQRS model for the transmission of malicious objects in computer network," Journal of Applied Mathematics, vol. 2014, Article ID 492198, 8 pages, 2014.

[16] L. Feng, X. Liao, H. Li, and Q. Han, "Hopf bifurcation analysis of a delayed viral infection model in computer networks," Mathematical and Computer Modelling, vol. 56, no. 7-8, pp. 167179, 2012.

[17] Y. Muroya, Y. Enatsu, and H. Li, "Global stability of a delayed SIRS computer virus propagation model," International Journal of Computer Mathematics, vol. 91, no. 3, pp. 347-367, 2014.

[18] J. G. Ren, X. F. Yang, L.-X. Yang, Y. H. Xu, and F. Z. Yang, "A delayed computer virus propagation model and its dynamics," Chaos, Solitons and Fractals, vol. 45, no. 1, pp. 74-79, 2012.

[19] L. Yang and X. Yang, "The effect of network topology on the spread of computer viruses: A Modelling Study," International Journal of Computer Mathematics, pp. 1-18, 2016.

[20] L.-X. Yang, X. Yang, and Y. Wu, "The impact of patch forwarding on the prevalence of computer virus: a theoretical assessment approach," Applied Mathematical Modelling, vol. 43, pp. 110-125, 2017.

[21] C. Gan, "Modeling and analysis of the effect of network eigenvalue on viral spread," Nonlinear Dynamics, vol. 84, no. 3 , pp. 1727-1733, 2016.

[22] C. Gan, X. Yang, W. Liu, and Q. Zhu, "A propagation model of computer virus with nonlinear vaccination probability," Communications in Nonlinear Science and Numerical Simulation, vol. 19, no. 1, pp. 92-100, 2014.

[23] C. Gan, X. Yang, and Q. Zhu, "Global stability of a computer virus propagation model with two kinds of generic nonlinear probabilities," Abstract and Applied Analysis, vol. 2014, Article ID 735327, 7 pages, 2014.

[24] M. Kumar, B. K. Mishra, and T. C. Panda, "Effect of quarantine \& vaccination on infectious nodes in computer network," International Journal of Computer Networks and Applications, vol. 2, pp. 92-97, 2015.

[25] B. D. Hassard, N. D. Kazarinoff, and Y. H. Wan, Theory and Applications of Hopf Bifurcation, vol. 41 of London Mathematical Society Lecture Note Series, Cambridge University Press, Cambridge, UK, 1981.

[26] X.-Y. Meng, H.-F. Huo, X.-B. Zhang, and H. Xiang, "Stability and Hopf bifurcation in a three-species system with feedback delays," Nonlinear Dynamics, vol. 64, no. 4, pp. 349-364, 2011.

[27] C. Xu, X. Tang, and M. Liao, "Stability and bifurcation analysis of a six-neuron BAM neural network model with discrete delays," Neurocomputing, vol. 74, no. 5, pp. 689-707, 2011.

[28] C. Bianca and L. Guerrini, "Existence of limit cycles in the Solow model with delayed-logistic population growth," The Scientific World Journal, vol. 2014, Article ID 207806, 2014.

[29] C. Xu, X. Tang, and M. Liao, "Stability and bifurcation analysis on a ring of five neurons with discrete delays," Journal of Dynamical and Control Systems, vol. 19, no. 2, pp. 237-275, 2013. 


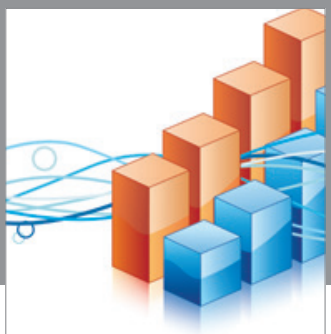

Advances in

Operations Research

vatem alat4

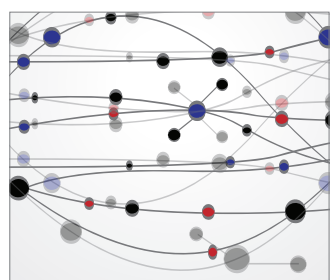

\section{The Scientific} World Journal
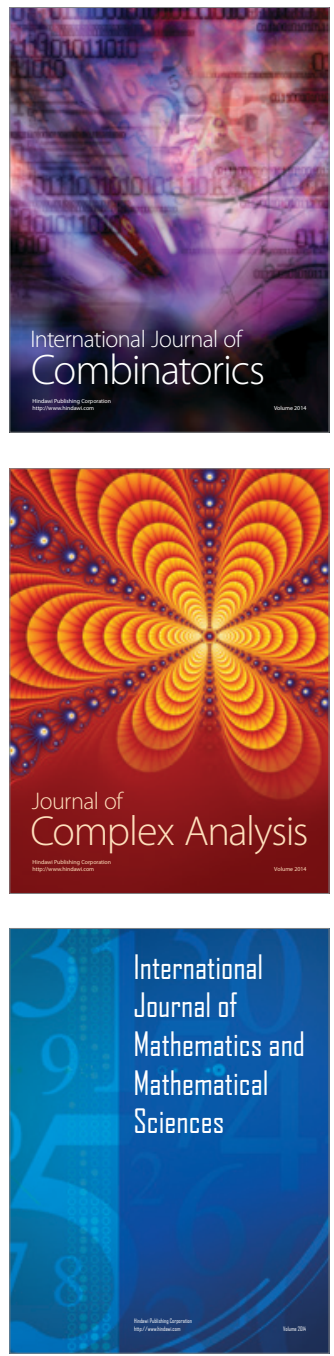
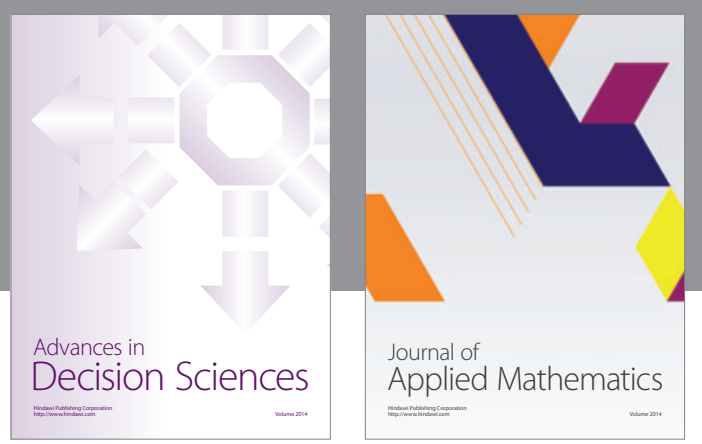

Algebra

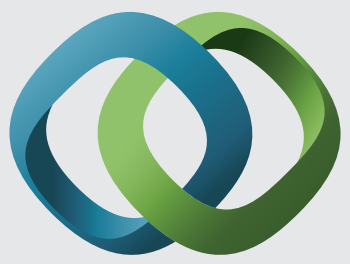

\section{Hindawi}

Submit your manuscripts at

https://www.hindawi.com
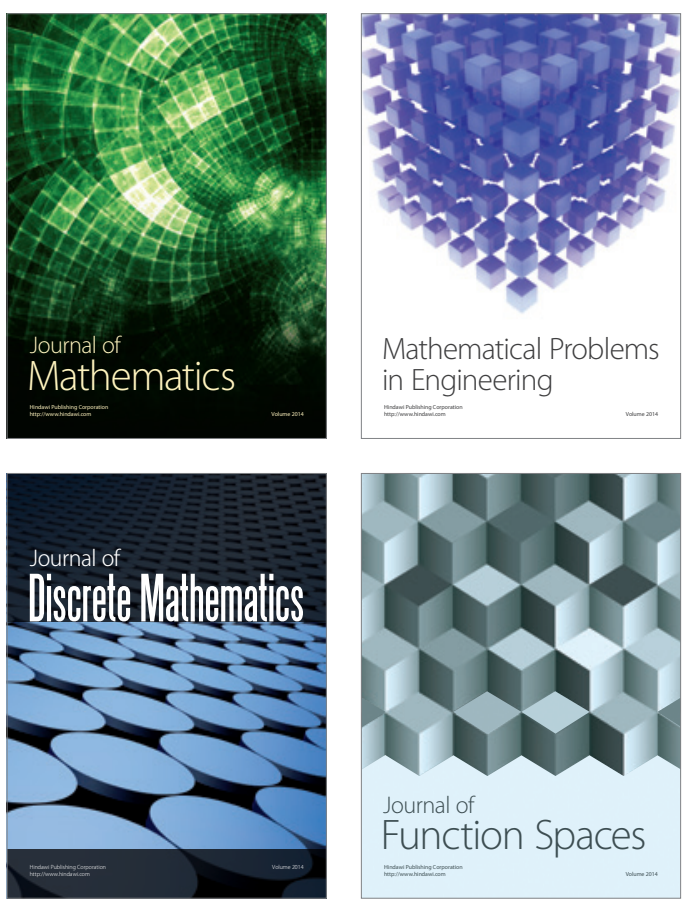

Mathematical Problems in Engineering
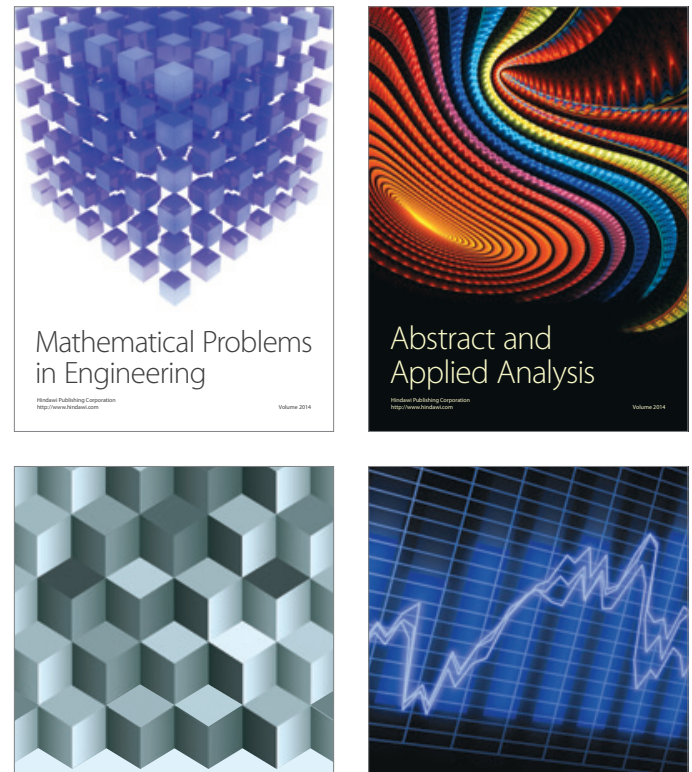

Journal of

Function Spaces

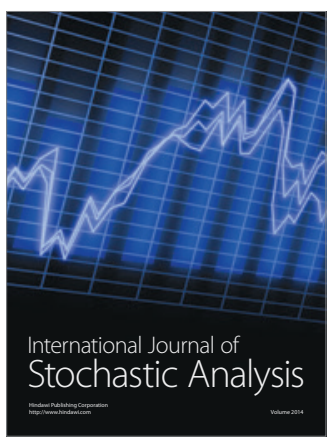

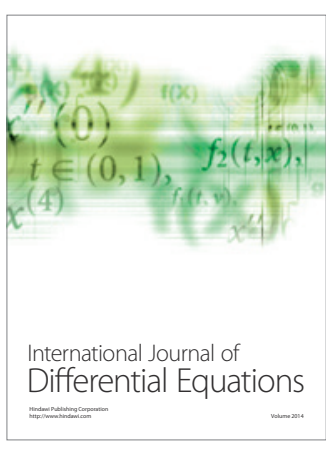
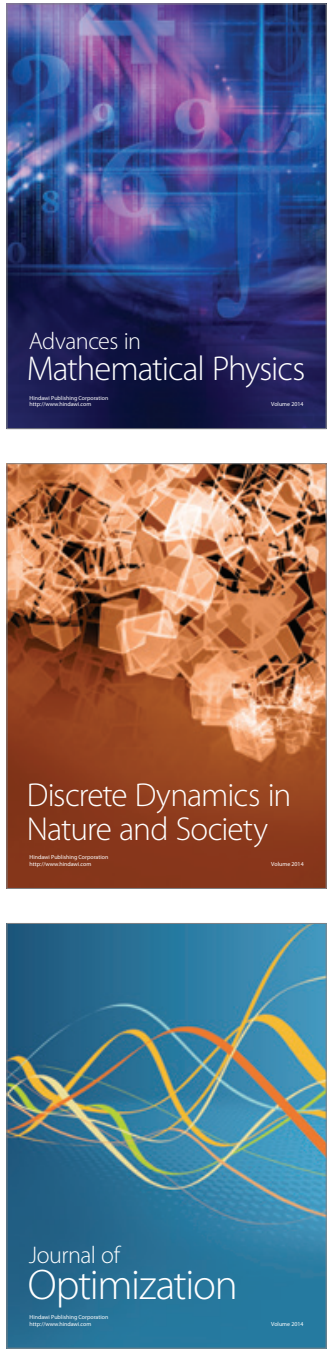